\title{
New Advances in the Modeling and Verification of Experimental Information for Ester-Alkane Solutions. Application to a Batch-Distillation Case
}

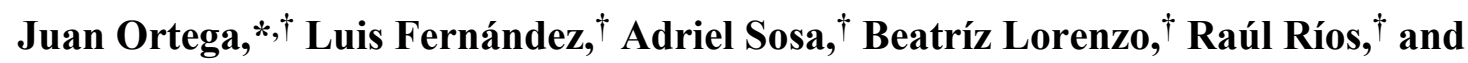
Jaime Wisniak ${ }^{\S}$

$\dagger$ Grupo de Ingeniería Térmica e Instrumentación (IDeTIC), Parque CientíficoTecnológico, Universidad de Las Palmas de Gran Canaria, 35071-Canary Islands, Spain

$\S$ Department of Chemical Engineering, Ben-Gurion University of the Negev, BeerSheva 84105 , Israel

* Corresponding author

E-mail address: juan.ortega@ulpgc.es 
Table S1. Background of published data for $v^{\mathrm{E}}, h^{\mathrm{E}}$ and iso-101.32kPa VLE, for the binaries: $\mathrm{H}_{5} \mathrm{C}_{2} \mathrm{COOC}_{v} \mathrm{H}_{2 v+1}(v=3,4)+\mathrm{C}_{n} \mathrm{H}_{2 n+2}(n=6-9)$, indicating the data serie from literature and those experimental measured for this work. *Values by other authors; ${ }^{+}$data set used only for comparison (non-used in modeling), ${ }^{\#}$ data set using in the modeling.

\begin{tabular}{|c|c|c|c|c|c|c|}
\hline & \multicolumn{3}{|c|}{ Propyl propanoate } & \multicolumn{3}{|c|}{ Butyl propanoate } \\
\hline & $v^{\mathrm{E}}$ & $h^{\mathrm{E}}$ & iso- $p$ VLE & $v^{\mathrm{E}}$ & $h^{\mathrm{E}}$ & iso- $p$ VLE \\
\hline $\begin{array}{l}\text { Hexane } \\
\text { (from } \\
\text { literature) }\end{array}$ & $\begin{array}{l}T=298 \\
\mathrm{~K}^{*,+, 1} \\
T=298 \mathrm{~K}^{+, 2}\end{array}$ & $\begin{array}{l}T=298 \mathrm{~K}^{\#, 2} \\
T=298 \mathrm{~K}^{*,+, 3} \\
T=308 \mathrm{~K}^{*, 3}\end{array}$ & & & & \\
\hline $\begin{array}{l}\text { Hexane } \\
\text { (this work) }\end{array}$ & $\begin{array}{l}T=288 \mathrm{~K}^{\#} \\
T=298 \mathrm{~K}^{\#} \\
T=308 \mathrm{~K}^{\#} \\
T=318 \mathrm{~K}^{\#} \\
T=328 \mathrm{~K}\end{array}$ & $T=318 \mathrm{~K}^{\#}$ & $p=101 \mathrm{kPa}^{\#}$ & $\begin{array}{l}T=288 \mathrm{~K}^{\#} \\
T=298 \mathrm{~K}^{\#} \\
T=308 \mathrm{~K}^{\#} \\
T=318 \mathrm{~K}^{\#} \\
T=328 \mathrm{~K}^{\#}\end{array}$ & $\begin{array}{l}T=298 \mathrm{~K}^{\#} \\
T=318 \mathrm{~K}^{\#}\end{array}$ & $p=101 \mathrm{kPa}^{\#}$ \\
\hline $\begin{array}{l}\text { Heptane } \\
\text { (from } \\
\text { literature) }\end{array}$ & $\begin{array}{l}T=298 \mathrm{~K}^{+, 4} \\
T=298 \mathrm{~K}^{+, 2} \\
T=298 \mathrm{~K}^{+, 5} \\
T=318 \mathrm{~K}^{+, 6}\end{array}$ & $\begin{array}{l}T=298 \mathrm{~K}^{\#, 2} \\
T=298 \mathrm{~K}^{\#, 5} \\
T=318 \mathrm{~K}^{6}\end{array}$ & $p=101 \mathrm{kPa}^{+, 4}$ & $\begin{array}{l}T=318 \mathrm{~K}^{+, 6} \\
T=298 \mathrm{~K}^{+, 7}\end{array}$ & $\begin{array}{l}T=318 \mathrm{~K}^{\#, 6} \\
T=298 \mathrm{~K}^{\#, 7}\end{array}$ & \\
\hline $\begin{array}{l}\text { Heptane } \\
\text { (this work) }\end{array}$ & $\begin{array}{l}T=288 \mathrm{~K}^{\#} \\
T=298 \mathrm{~K}^{\#} \\
T=308 \mathrm{~K}^{\#} \\
T=318 \mathrm{~K}^{\#} \\
T=328 \mathrm{~K}^{\#}\end{array}$ & & $p=101 \mathrm{kPa}^{\#}$ & $\begin{array}{l}T=288 \mathrm{~K}^{\#} \\
T=298 \mathrm{~K}^{\#} \\
T=308 \mathrm{~K}^{\#} \\
T=318 \mathrm{~K}^{\#} \\
T=328 \mathrm{~K}^{\#}\end{array}$ & & $p=101 \mathrm{kPa}^{\#}$ \\
\hline $\begin{array}{l}\text { Octane } \\
\text { (from } \\
\text { literature) } \\
\end{array}$ & $T=298 \mathrm{~K}^{+, 2}$ & $T=298 \mathrm{~K}^{\#, 2}$ & & & & \\
\hline $\begin{array}{l}\text { Octane } \\
\text { (this work) }\end{array}$ & $\begin{array}{l}T=288 \mathrm{~K}^{\#} \\
T=298 \mathrm{~K}^{\#} \\
T=308 \mathrm{~K}^{\#} \\
T=318 \mathrm{~K}^{\#} \\
T=328 \mathrm{~K}^{\#}\end{array}$ & $T=318 \mathrm{~K}^{\#}$ & $p=101 \mathrm{kPa}^{\#}$ & $\begin{array}{l}T=288 \mathrm{~K}^{\#} \\
T=298 \mathrm{~K}^{\#} \\
T=308 \mathrm{~K}^{\#} \\
T=318 \mathrm{~K}^{\#} \\
T=328 \mathrm{~K}^{\#}\end{array}$ & $\begin{array}{l}T=298 \mathrm{~K}^{\#} \\
T=318 \mathrm{~K}^{\#}\end{array}$ & $p=101 \mathrm{kPa}^{\#}$ \\
\hline $\begin{array}{l}\text { Nonane } \\
\text { (from } \\
\text { literature) }\end{array}$ & $\begin{array}{l}T=298 \mathrm{~K}^{+, 4} \\
T=298 \mathrm{~K}^{+, 2} \\
T=298 \mathrm{~K}^{+, 5} \\
T=318 \mathrm{~K}^{+, 6}\end{array}$ & $\begin{array}{l}T=298 \mathrm{~K}^{\#, 2} \\
T=298 \mathrm{~K}^{\#, 5} \\
T=318 \mathrm{~K}^{\#, 6}\end{array}$ & $p=101 \mathrm{kPa}^{+, 4}$ & $\begin{array}{l}T=318 \mathrm{~K}^{+, 6} \\
T=298 \mathrm{~K}^{+, 7}\end{array}$ & $\begin{array}{l}T=318 \mathrm{~K}^{\#, 6} \\
T=298 \mathrm{~K}^{\#, 7}\end{array}$ & \\
\hline $\begin{array}{l}\text { Nonane } \\
\text { (this work) }\end{array}$ & $\begin{array}{l}T=288 \mathrm{~K}^{\#} \\
T=298 \mathrm{~K}^{\#} \\
T=308 \mathrm{~K}^{\#} \\
T=318 \mathrm{~K}^{\#} \\
T=328 \mathrm{~K}^{\#}\end{array}$ & & $p=101 \mathrm{kPa}^{\#}$ & $\begin{array}{l}T=288 \mathrm{~K}^{\#} \\
T=298 \mathrm{~K}^{\#} \\
T=308 \mathrm{~K}^{\#} \\
T=318 \mathrm{~K}^{\#} \\
T=328 \mathrm{~K}^{\#}\end{array}$ & & $p=101 \mathrm{kPa}^{\#}$ \\
\hline
\end{tabular}

[1] Casas, H.; Segade, L.; Franjo, C.; Jiménez, E.; Paz Andrade, M. I. Excess Properties for Propyl Propanoate + Hexane + Benzene at 298.15 K. J. Chem. Eng. Data 1998, 43 (5), 756762.

[2] Lorenzana, M. T.; Legido, J. L.; Jimenez, E.; Fernandez, J.; Pias, L.; Ortega, J.; Andrade, M. I. P. Thermodynamic Properties of (a Propyl Ester + an n-Alkane) at $298.15 \mathrm{~K}$ I. $\left\{x \mathrm{C}_{2} \mathrm{H}_{5} \mathrm{CO}_{2} \mathrm{C}_{3} \mathrm{H}_{7}+(1-x) \mathrm{C}_{\mathrm{n}} \mathrm{H}_{2 \mathrm{n}+2}\right\},(\mathrm{n}=6$ to 10). J. Chem. Thermodyn. 1989, 21, 1017-1022.

[3] Casas, H.; Segade, L.; Franjo, C.; Jiménez, E.; Paz Andrade, M. I. Excess Molar Enthalpies of Propyl Propanoate+ Hexane+ Benzene at 298.15 K and 308.15 K. J. Chem. Eng. Data 2000, 45 (3), 445-449.

[4] Ortega, J.; Gonzalez, C.; Galvan, S. Vapor-Liquid Equilibria for Binary Systems Composed 
of a Propyl Ester (Ethanoate, Propanoate, Butanoate) + an n-Alkane $\left(\mathrm{C}_{7}, \mathrm{C}_{9}\right) . J$. Chem. Eng. Data 2001, 46, 904-912.

[5] Ortega, J.; Vidal, M.; Toledo, F. J.; Placido, J. Thermodynamic Properties of (a Propyl Ester + an n-Alkane). XII. Excess Molar Enthalpies and Excess Molar Volumes for $\left\{x \mathrm{CH}_{3}\left(\mathrm{CH}_{2}\right)_{\mathrm{u}-}\right.$ $\left.{ }_{1} \mathrm{COO}\left(\mathrm{CH}_{2}\right)_{2} \mathrm{CH}_{3}+(1-x) \mathrm{CH}_{3}\left(\mathrm{CH}_{2}\right)_{2 \mathrm{v}+1} \mathrm{CH}_{3}\right\}$ with $\mathrm{u}=(1$ to 3$)$, and $\mathrm{v}=(1$ to 7$)$. J. Chem. Thermodyn. 1999, 31, 1025-1044.

[6] Ortega, J.; Espiau, F.; Toledo, F. J.; Dieppa, R. Thermodynamic Properties of (an Ester + an Alkane). XVII. Experimental $\mathrm{H}_{\mathrm{m}}^{\mathrm{E}}$ and $\mathrm{V}_{\mathrm{m}}^{\mathrm{E}}$ Values for (an Alkyl Propanoate + an Alkane) at 318.15 K. J. Chem. Thermodyn. 2005, 37, 967-983.

[7] Toledo, F. J.; Ortega, J.; Chaar, M.; Vidal, M. Thermodynamic Properties of (a Butyl Ester + an-Alkane). XIII. $\mathrm{H}_{\mathrm{m}}^{\mathrm{E}}$ and $\mathrm{V}_{\mathrm{m}}^{\mathrm{E}}$ for $\left\{x \mathrm{CH}_{3}\left(\mathrm{CH}_{2}\right)_{\mathrm{u}-1} \mathrm{CO}_{2}\left(\mathrm{CH}_{2}\right)_{3}+(1-x) \mathrm{CH}_{3}\left(\mathrm{CH}_{2}\right)_{2 \mathrm{v}+1} \mathrm{CH}_{3}\right\}$, Where $\mathrm{u}=1$ to 3 and $\mathrm{v}=1$ to 7 . J. Chem. Thermodyn. 2000, 32, 1013-1036. 
Table S2. Experimental values obtained for densities $\rho$, refractive indices $n_{\mathrm{D}}$, and isobaric expansivities $\alpha$, obtained at $298.15 \mathrm{~K}$ and atmospheric pressure $(p \approx 98.9 \mathrm{kPa})$, and normal boiling temperature $T_{\mathrm{b}, \mathrm{i}}^{\mathrm{o}}$, of pure compounds.

\begin{tabular}{|c|c|c|c|c|c|c|c|c|}
\hline \multirow{2}{*}{ Compound } & \multicolumn{2}{|c|}{$T_{\mathrm{b}, \mathrm{i}}^{0} / \mathrm{K}$} & \multicolumn{2}{|c|}{$\rho / \mathrm{kgm}^{-3}$} & \multicolumn{2}{|l|}{$n_{\mathrm{D}}$} & \multicolumn{2}{|c|}{$10^{3} \cdot \alpha / \mathrm{K}^{-1}$} \\
\hline & exp. & lit. & exp. & lit. & exp. & lit. & exp. & lit. \\
\hline propyl propanoate & $395.32 \pm 0.09$ & $\begin{array}{l}395.65^{17} \\
395.30^{18}\end{array}$ & $875.67 \pm 0.01$ & $\begin{array}{l}876.70^{17} \\
875.65^{21}\end{array}$ & $1.3912 \pm 2 \cdot 10^{-5}$ & $\begin{array}{l}1.3920^{17} \\
1.3918^{21}\end{array}$ & 1.24 & $1.28^{17}$ \\
\hline butyl propanoate & $418.70 \pm 0.03$ & $\begin{array}{l}419.75^{17} \\
418.69^{19}\end{array}$ & $871.18 \pm 0.01$ & $\begin{array}{l}871.40^{17} \\
870.88^{19}\end{array}$ & $1.3992 \pm 2 \cdot 10^{-5}$ & $\begin{array}{l}1.4000^{17} \\
1.3992^{19}\end{array}$ & 1.15 & $1.14^{17}$ \\
\hline hexane & $341.82 \pm 0.02$ & $341.89^{16}$ & $654.87 \pm 0.05$ & $\begin{array}{l}654.81^{16} \\
654.82^{22}\end{array}$ & $1.3725 \pm 5 \cdot 10^{-5}$ & $\begin{array}{l}1.3723^{16} \\
1.3725^{22}\end{array}$ & 1.44 & $1.39^{16}$ \\
\hline heptane & $371.38 \pm 0.06$ & $\begin{array}{l}371.35^{20} \\
371.58^{16}\end{array}$ & $679.62 \pm 0.03$ & $\begin{array}{l}679.60^{20} \\
679.51^{16}\end{array}$ & $1.3855 \pm 3 \cdot 10^{-5}$ & $\begin{array}{c}1.3853^{20} \\
1.38511^{16}\end{array}$ & 1.29 & $1.25^{17}$ \\
\hline octane & $398.82 \pm 0.07$ & $\begin{array}{l}398.81^{16} \\
398.82^{17}\end{array}$ & $698.55 \pm 0.03$ & $\begin{array}{l}698.49^{16} \\
698.44^{22}\end{array}$ & $1.3957 \pm 3 \cdot 10^{-5}$ & $1.3955^{22}$ & 1.19 & $1.23^{17}$ \\
\hline nonane & $423.90 \pm 0.09$ & $\begin{array}{l}423.95^{16} \\
423.97^{17}\end{array}$ & $713.85 \pm 0.01$ & $\begin{array}{l}713.81^{16} \\
713.75^{17}\end{array}$ & $1.4039 \pm 1 \cdot 10^{-5}$ & $1.43011^{16}$ & 1.12 & $1.19^{17}$ \\
\hline
\end{tabular}

[16] Riddick, J.A.; Bunger, W.B.; Sakano, T.K. Organic Solvents. Physical Properties and Methods of Purification 4th Ed, Wiley-Interscience, NY. 1986.

[17] TRC. Thermodynamic Tables Non-Hydrocarbons \& Hydrocarbons; Thermodynamic Research Center. Texas A\&M University System: College Station. TX. 1965.

[18] Fárková. J.; Wichterle. I. Vapour pressures of some ethyl and propyl esters of fatty acids Fluid Phase Equilib. 1993. 90, 143-148.

[19] Muñoz. R.; Montón. J.B.; Burguet. M.C.; de la Torre. J. Vapor-liquid equilibria in the ternary system isobutyl alcohol+isobutyl acetate+ butyl propionate and the binary systems isobutyl alcohol+ butyl propionate. isobutyl acetate+ butyl propionate at $101.3 \mathrm{kPa}$. Fluid Phase Equilib. 2005, 238, 65-71.

[20] Ortega, J.; Espiau, F.; Tojo, J.; Canosa, J.; Rodríguez, A. Isobaric Vapor- Liquid Equilibria and Excess Properties for the Binary Systems of Methyl Esters+ Heptane. J. Chem. Eng. Data, 2003, 48(5), 1183-1190.

[21] Ortega. J.; Galvan. S. Vapor-liquid-equilibria of propyl propanoate with 1-alkanols at 101.32 KPa of pressure. J. Chem. Eng. Data 1994, 39(4). 907-910. [22] Ortega. J.; Matos. J.S. Estimation of the isobaric expansivities from several equations of molar refraction for some pure organic compounds. Mat. Chem. Phys. 1986, 15, 415-425. 
Table S3. Experimental vapor pressure for alkyl propanoates

\begin{tabular}{|c|c|c|c|c|c|c|c|c|c|}
\hline$T / \mathrm{K}$ & $p_{\mathrm{i}}^{\circ} / \mathrm{kPa}$ & $T / \mathrm{K}$ & $p_{\mathrm{i}}^{\mathrm{o}} / \mathrm{kPa}$ & $T / \mathrm{K}$ & $p_{\mathrm{i}}^{\circ} / \mathrm{kPa}$ & $T / \mathrm{K}$ & $p_{\mathrm{i}}^{\mathrm{o}} / \mathrm{kPa}$ & $T / \mathrm{K}$ & $p_{\mathrm{i}}^{\circ} / \mathrm{kPa}$ \\
\hline \multicolumn{10}{|c|}{ propyl propanoate } \\
\hline 364.98 & 37.50 & 383.53 & 70.00 & 395.72 & 101.32 & 413.32 & 165.00 & 426.44 & 230.00 \\
\hline 366.78 & 40.00 & 384.65 & 72.50 & 396.95 & 105.00 & 414.47 & 170.00 & 427.33 & 235.00 \\
\hline 368.50 & 42.50 & 385.74 & 75.00 & 398.55 & 110.00 & 415.58 & 175.00 & 428.20 & 240.00 \\
\hline 370.16 & 45.00 & 386.78 & 77.50 & 400.10 & 115.00 & 416.67 & 180.00 & 429.05 & 245.00 \\
\hline 371.73 & 47.50 & 387.80 & 80.00 & 401.59 & 120.00 & 417.73 & 185.00 & 429.90 & 250.00 \\
\hline 373.28 & 50.00 & 388.81 & 82.50 & 403.06 & 125.00 & 418.78 & 190.00 & 430.73 & 255.00 \\
\hline 374.71 & 52.50 & 389.77 & 85.00 & 404.48 & 130.00 & 419.80 & 195.00 & 431.54 & 260.00 \\
\hline 376.07 & 55.00 & 390.75 & 87.50 & 405.83 & 135.00 & 420.81 & 200.00 & 432.34 & 265.00 \\
\hline 377.42 & 57.50 & 391.65 & 90.00 & 407.18 & 140.00 & 421.79 & 205.00 & 433.14 & 270.00 \\
\hline 378.74 & 60.00 & 392.59 & 92.50 & 408.47 & 145.00 & 422.75 & 210.00 & 433.92 & 275.00 \\
\hline 379.99 & 62.50 & 393.49 & 95.00 & 409.73 & 150.00 & 423.69 & 215.00 & 434.69 & 280.00 \\
\hline 381.21 & 65.00 & 394.37 & 97.50 & 410.96 & 155.00 & 424.62 & 220.00 & & \\
\hline 382.39 & 67.50 & 395.29 & 100.00 & 412.16 & 160.00 & 425.54 & 225.00 & & \\
\hline \multicolumn{10}{|c|}{ butyl propanoate } \\
\hline 389.91 & 42.50 & 406.65 & 72.50 & 418.62 & 101.32 & 435.89 & 160.00 & 448.94 & 220.00 \\
\hline 391.67 & 45.00 & 407.83 & 75.00 & 419.89 & 105.00 & 437.12 & 165.00 & 449.93 & 225.00 \\
\hline 393.34 & 47.50 & 408.90 & 77.50 & 421.64 & 110.00 & 438.31 & 170.00 & 450.87 & 230.00 \\
\hline 394.88 & 50.00 & 409.93 & 80.00 & 423.33 & 115.00 & 439.46 & 175.00 & 451.78 & 235.00 \\
\hline 396.28 & 52.50 & 411.01 & 82.50 & 424.92 & 120.00 & 440.61 & 180.00 & 452.72 & 240.00 \\
\hline 397.68 & 55.00 & 411.99 & 85.00 & 426.44 & 125.00 & 441.69 & 185.00 & 453.18 & 245.00 \\
\hline 399.11 & 57.50 & 412.93 & 87.50 & 427.96 & 130.00 & 442.66 & 190.00 & 454.16 & 250.00 \\
\hline 400.46 & 60.00 & 413.99 & 90.00 & 429.41 & 135.00 & 443.79 & 195.00 & 454.95 & 255.00 \\
\hline 401.74 & 62.50 & 414.96 & 92.50 & 430.80 & 140.00 & 444.84 & 200.00 & 455.87 & 260.00 \\
\hline 402.94 & 65.00 & 415.88 & 95.00 & 432.24 & 145.00 & 445.93 & 205.00 & 456.73 & 265.00 \\
\hline 404.28 & 67.50 & 416.84 & 97.50 & 433.49 & 150.00 & 446.92 & 210.00 & 457.59 & 270.00 \\
\hline 405.46 & 70.00 & 417.78 & 100.00 & 434.61 & 155.00 & 447.94 & 215.00 & & \\
\hline
\end{tabular}


Table S4. Values of $\left(\rho / \mathrm{kg} \cdot \mathrm{m}^{-3}, p / \mathrm{MPa}, T / \mathrm{K}\right)$ for pure compounds measured at different pressures and temperatures

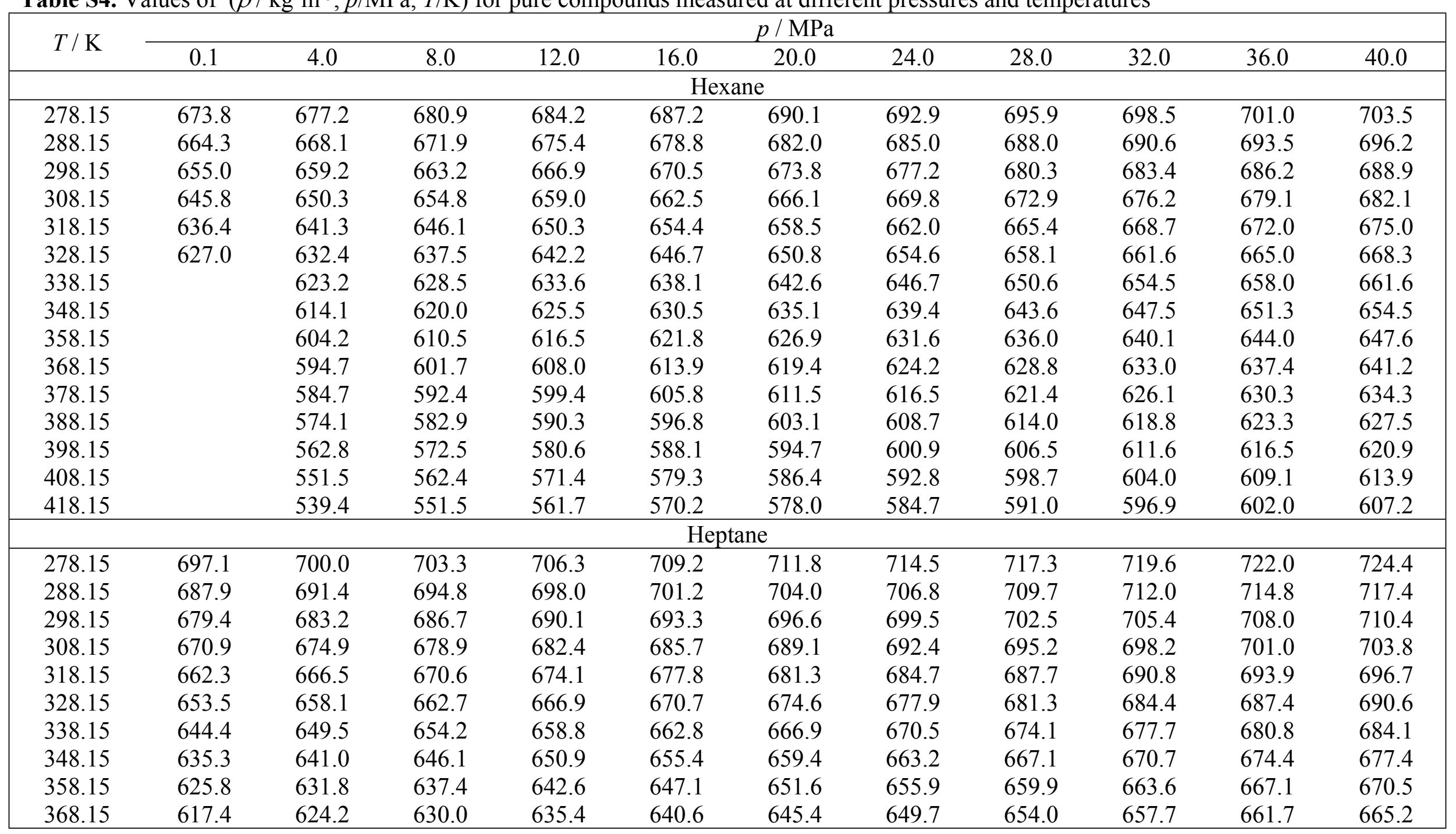




\begin{tabular}{|c|c|c|c|c|c|c|c|c|c|c|c|}
\hline 378.15 & & 615.1 & 621.6 & 627.5 & 632.9 & 638.0 & 642.4 & 646.9 & 651.1 & 654.8 & 658.6 \\
\hline 388.15 & & 605.8 & 612.8 & 619.1 & 624.8 & 630.2 & 635.3 & 639.9 & 644.3 & 648.5 & 652.3 \\
\hline 398.15 & & 595.9 & 603.5 & 610.4 & 616.7 & 622.5 & 627.7 & 632.6 & 637.1 & 641.6 & 645.6 \\
\hline 408.15 & & 586.0 & 594.4 & 602.0 & 608.6 & 614.7 & 620.4 & 625.6 & 630.3 & 634.9 & 639.3 \\
\hline 418.15 & & 575.4 & 584.9 & 593.0 & 600.2 & 606.8 & 612.8 & 618.2 & 623.6 & 628.4 & 633.0 \\
\hline \multicolumn{12}{|c|}{ Octane } \\
\hline 278.15 & 715.5 & 718.4 & 721.4 & 724.1 & 726.8 & 729.3 & 731.7 & 734.4 & 736.6 & 738.8 & 741.0 \\
\hline 288.15 & 706.6 & 709.9 & 712.9 & 715.9 & 718.9 & 721.5 & 724.1 & 726.7 & 729.0 & 731.7 & 734.1 \\
\hline 298.15 & 698.3 & 701.7 & 705.1 & 708.3 & 711.2 & 714.2 & 717.1 & 719.8 & 722.4 & 724.9 & 727.3 \\
\hline 308.15 & 690.1 & 694.0 & 697.7 & 701.0 & 704.0 & 707.1 & 710.3 & 713.0 & 715.8 & 718.3 & 721.1 \\
\hline 318.15 & 682.1 & 685.9 & 689.8 & 693.3 & 696.7 & 700.1 & 703.3 & 706.0 & 709.0 & 711.9 & 714.5 \\
\hline 328.15 & 673.8 & 678.1 & 682.1 & 686.0 & 689.7 & 693.1 & 696.4 & 699.5 & 702.5 & 705.5 & 708.4 \\
\hline 338.15 & 665.5 & 670.1 & 674.3 & 678.4 & 682.0 & 685.8 & 689.1 & 692.5 & 695.9 & 698.8 & 701.9 \\
\hline 348.15 & 657.1 & 662.0 & 666.7 & 671.0 & 674.9 & 678.7 & 682.2 & 685.9 & 689.3 & 692.7 & 695.4 \\
\hline 358.15 & 648.6 & 653.9 & 658.8 & 663.5 & 667.6 & 671.6 & 675.4 & 679.3 & 682.7 & 686.1 & 689.2 \\
\hline 368.15 & 640.0 & 646.0 & 651.0 & 656.0 & 660.6 & 664.9 & 668.9 & 672.6 & 676.2 & 680.0 & 683.3 \\
\hline 378.15 & & 637.3 & 642.9 & 648.2 & 653.2 & 657.8 & 661.9 & 666.2 & 669.9 & 673.3 & 677.1 \\
\hline 388.15 & & 628.5 & 634.7 & 640.2 & 645.3 & 650.4 & 654.8 & 659.0 & 663.0 & 666.9 & 670.3 \\
\hline 398.15 & & 619.5 & 625.9 & 631.9 & 637.6 & 643.0 & 647.6 & 652.1 & 656.3 & 660.2 & 664.0 \\
\hline 408.15 & & 610.1 & 617.3 & 623.7 & 629.6 & 635.1 & 640.1 & 644.9 & 649.3 & 653.5 & 657.7 \\
\hline 418.15 & & 600.6 & 608.4 & 615.3 & 621.7 & 627.6 & 632.8 & 638.1 & 642.8 & 647.1 & 651.4 \\
\hline \multicolumn{12}{|c|}{ Nonane } \\
\hline 278.15 & 729.9 & 732.7 & 735.6 & 738.3 & 740.7 & 743.1 & 745.5 & 748.0 & 750.1 & 752.3 & 754.4 \\
\hline 288.15 & 721.5 & 724.4 & 727.4 & 730.3 & 733.0 & 735.6 & 738.0 & 740.6 & 742.8 & 745.3 & 747.6 \\
\hline 298.15 & 713.4 & 716.7 & 719.8 & 722.8 & 725.6 & 728.4 & 731.1 & 733.8 & 736.4 & 738.6 & 740.9 \\
\hline 308.15 & 705.6 & 709.0 & 712.6 & 715.9 & 718.5 & 721.6 & 724.6 & 727.1 & 729.8 & 732.2 & 734.8 \\
\hline 318.15 & 697.7 & 701.5 & 705.2 & 708.5 & 711.6 & 715.0 & 717.8 & 720.5 & 723.6 & 726.1 & 728.6 \\
\hline 328.15 & 689.9 & 694.0 & 697.8 & 701.3 & 704.9 & 708.1 & 711.0 & 714.0 & 716.9 & 719.6 & 722.6 \\
\hline 338.15 & 682.0 & 686.4 & 690.2 & 694.0 & 697.4 & 701.0 & 704.1 & 707.4 & 710.5 & 713.2 & 716.1 \\
\hline 348.15 & 673.8 & 678.4 & 682.7 & 686.8 & 690.4 & 694.0 & 697.5 & 700.7 & 704.0 & 707.3 & 709.8 \\
\hline
\end{tabular}




\begin{tabular}{|c|c|c|c|c|c|c|c|c|c|c|c|}
\hline 358.15 & 666.0 & 670.8 & 675.4 & 679.6 & 683.5 & 687.3 & 690.9 & 694.3 & 697.6 & 700.8 & 703.7 \\
\hline 368.15 & 658.0 & 663.3 & 668.1 & 672.5 & 676.8 & 680.9 & 684.6 & 688.1 & 691.4 & 695.0 & 698.2 \\
\hline 388.15 & & 646.9 & 652.7 & 657.6 & 662.2 & 666.8 & 671.0 & 675.0 & 678.9 & 682.4 & 685.7 \\
\hline 408.15 & & 629.8 & 636.2 & 642.1 & 647.5 & 652.6 & 657.3 & 661.6 & 665.6 & 669.6 & 673.4 \\
\hline 418.15 & & 620.7 & 627.9 & 634.2 & 639.9 & 645.4 & 650.3 & 655.0 & 659.2 & 663.2 & 667.5 \\
\hline \multicolumn{12}{|c|}{ Propyl propanoate } \\
\hline 298.15 & 875.0 & 878.7 & 882.1 & 885.5 & 888.8 & 892.0 & 895.1 & 898.1 & 901.1 & 903.8 & 906.5 \\
\hline 308.15 & 864.2 & 868.0 & 871.9 & 875.5 & 878.8 & 882.3 & 885.8 & 888.5 & 891.7 & 894.5 & 897.4 \\
\hline 318.15 & 853.7 & 857.7 & 862.0 & 865.6 & 869.3 & 873.0 & 876.5 & 879.5 & 882.7 & 885.8 & 888.8 \\
\hline 328.15 & 843.1 & 847.6 & 852.0 & 856.0 & 859.9 & 863.7 & 867.2 & 870.6 & 874.0 & 877.2 & 880.5 \\
\hline 338.15 & 832.4 & 837.3 & 841.7 & 846.1 & 850.0 & 854.1 & 857.7 & 861.4 & 865.2 & 868.3 & 871.7 \\
\hline 348.15 & 821.5 & 826.7 & 831.6 & 836.3 & 840.5 & 844.7 & 848.6 & 852.5 & 856.3 & 860.0 & 863.3 \\
\hline 408.15 & & 759.0 & 766.7 & 773.7 & 780.4 & 786.5 & 792.4 & 797.4 & 802.5 & 807.3 & 812.0 \\
\hline 418.15 & & 746.7 & 755.2 & 762.8 & 769.7 & 776.4 & 782.2 & 787.6 & 793.5 & 798.4 & 803.2 \\
\hline \multicolumn{12}{|c|}{ Butyl propanoate } \\
\hline 278.15 & 891.5 & 894.4 & 897.5 & 900.4 & 903.0 & 905.6 & 908.3 & 910.9 & 913.3 & 915.7 & 918.0 \\
\hline 288.15 & 880.9 & 883.8 & 887.2 & 890.3 & 893.2 & 896.1 & 898.7 & 901.4 & 903.8 & 906.6 & 909.1 \\
\hline 298.15 & 870.6 & 874.1 & 877.5 & 880.6 & 883.7 & 886.6 & 889.4 & 892.3 & 895.1 & 897.7 & 900.2 \\
\hline 308.15 & 860.7 & 864.3 & 868.2 & 871.4 & 874.6 & 877.9 & 880.9 & 883.8 & 886.8 & 889.5 & 892.2 \\
\hline 318.15 & 850.5 & 854.4 & 858.3 & 861.8 & 865.3 & 869.0 & 872.1 & 875.1 & 878.1 & 881.2 & 883.9 \\
\hline 328.15 & 840.9 & 844.8 & 848.9 & 852.8 & 856.5 & 860.0 & 863.3 & 866.5 & 869.6 & 872.8 & 875.9 \\
\hline
\end{tabular}




\begin{tabular}{|c|c|c|c|c|c|c|c|c|c|c|c|}
\hline 338.15 & 830.9 & 835.3 & 839.5 & 843.6 & 847.3 & 851.1 & 854.6 & 857.9 & 861.5 & 864.5 & 867.8 \\
\hline 348.15 & 820.8 & 825.4 & 830.0 & 834.5 & 838.4 & 842.4 & 846.0 & 849.7 & 853.3 & 856.7 & 859.9 \\
\hline 368.15 & 800.4 & 806.1 & 811.0 & 815.9 & 820.5 & 825.0 & 829.0 & 833.0 & 836.8 & 840.7 & 844.2 \\
\hline 388.15 & & 785.4 & 791.4 & 796.7 & 801.9 & 806.8 & 811.5 & 815.9 & 820.2 & 824.1 & 828.0 \\
\hline 398.15 & & 774.7 & 781.0 & 786.8 & 792.5 & 797.8 & 802.7 & 807.2 & 811.6 & 815.9 & 819.8 \\
\hline 408.15 & & 763.6 & 770.5 & 776.8 & 782.7 & 788.2 & 793.6 & 798.5 & 803.0 & 807.4 & 811.7 \\
\hline
\end{tabular}


Table S5. Parameters of the Tait equation, Eqs 4 and 5 Obtained in the Modeling of $(p, \rho, T)$ Values

\begin{tabular}{|ccccccc|}
\hline & Hexane & heptane & octane & nonane & $\begin{array}{c}\text { propyl } \\
\text { propanoate }\end{array}$ & $\begin{array}{c}\text { butyl } \\
\text { propanoate }\end{array}$ \\
\hline$A_{0}$ & $9.84 \mathrm{E} 2$ & $8.15 \mathrm{E} 2$ & $8.57 \mathrm{E} 2$ & $8.73 \mathrm{E} 2$ & $1.11 \mathrm{E} 3$ & $1.09 \mathrm{E} 3$ \\
$A_{1}$ & -1.21 & $-7.69 \mathrm{E}-2$ & $-2.67 \mathrm{E}-1$ & $-3.05 \mathrm{E}-1$ & $-4.98 \mathrm{E}-1$ & $-4.78 \mathrm{E}-1$ \\
$A_{2}$ & $4.01 \mathrm{E}-4$ & $-1.26 \mathrm{E}-3$ & $-8.80 \mathrm{E}-4$ & $-7.67 \mathrm{E}-4$ & $-9.10 \mathrm{E}-4$ & $-8.33 \mathrm{E}-4$ \\
$B_{0}$ & $1.20 \mathrm{E} 2$ & $2.19 \mathrm{E} 2$ & $2.38 \mathrm{E} 2$ & $2.44 \mathrm{E} 2$ & $2.86 \mathrm{E} 2$ & $3.00 \mathrm{E} 2$ \\
$B_{1}$ & $-5.58 \mathrm{E}-1$ & $-5.04 \mathrm{E}-1$ & $-5.36 \mathrm{E}-1$ & $-5.40 \mathrm{E}-1$ & $-6.41 \mathrm{E}-1$ & $-6.62 \mathrm{E}-1$ \\
$B_{2}$ & -4.02 & $-4.68 \mathrm{E}-6$ & $-1.95 \mathrm{E}-6$ & $-6.43 \mathrm{E}-7$ & $-1.93 \mathrm{E}-6$ & $-8.73 \mathrm{E}-7$ \\
$C_{0}$ & $8.96 \mathrm{E}-2$ & $9.44 \mathrm{E}-2$ & $9.64 \mathrm{E}-2$ & $9.41 \mathrm{E}-2$ & $9.81 \mathrm{E}-2$ & $9.97 \mathrm{E}-2$ \\
$C_{1}$ & 5.68 & $2.53 \mathrm{E}-6$ & $1.72 \mathrm{E}-6$ & $7.29 \mathrm{E}-7$ & $1.97 \mathrm{E}-6$ & $1.09 \mathrm{E}-6$ \\
$C_{2}$ & $-2.39 \mathrm{E}-2$ & $-1.35 \mathrm{E}-7$ & $-9.70 \mathrm{E}-8$ & $-6.49 \mathrm{E}-8$ & $-1.01 \mathrm{E}-7$ & $-7.61 \mathrm{E}-8$ \\
$A R D$ & 0.273 & 0.650 & 0.406 & 0.284 & 0.353 & 0.250 \\
$R M S E$ & 2.290 & 5.357 & 3.383 & 2.438 & 3.677 & 2.678 \\
\hline
\end{tabular}


Table S6. Densities $\rho$, and excess molar volumes $v^{\mathrm{E}}$, for the binaries an alkyl propanoate $(1)+$ an alkane $(2)$ at several temperatures and atmospheric pressure $(p \approx 98.9$ $\mathrm{kPa})$.

\begin{tabular}{|c|c|c|c|c|c|c|c|c|}
\hline$x_{1}$ & $\frac{\rho}{\mathrm{kg} \cdot \mathrm{m}^{-3}}$ & $\frac{10^{9} \cdot v^{\mathrm{E}}}{\mathrm{m}^{3} \cdot \mathrm{mol}^{-1}}$ & $x_{1}$ & $\frac{\rho}{\mathrm{kg} \cdot \mathrm{m}^{-3}}$ & $\frac{10^{9} \cdot v^{\mathrm{E}}}{\mathrm{m}^{3} \cdot \mathrm{mol}^{-1}}$ & $x_{1}$ & $\frac{\rho}{\mathrm{kg} \cdot \mathrm{m}^{-3}}$ & $\frac{10^{9} \cdot v^{\mathrm{E}}}{\mathrm{m}^{3} \cdot \mathrm{mol}^{-1}}$ \\
\hline \multicolumn{9}{|c|}{ propyl propanoate (1)+hexane (2) } \\
\hline \multicolumn{9}{|c|}{$288.15 \mathrm{~K}$} \\
\hline 0.0000 & 664.00 & 0 & 0.3445 & 739.08 & 352 & 0.6935 & 817.12 & 236 \\
\hline 0.0478 & 674.19 & 102 & 0.3922 & 749.67 & 351 & 0.7459 & 828.90 & 206 \\
\hline 0.0975 & 684.90 & 184 & 0.4441 & 761.24 & 344 & 0.7954 & 840.12 & 161 \\
\hline 0.1490 & 696.12 & 237 & 0.4948 & 772.55 & 331 & 0.8480 & 851.91 & 131 \\
\hline 0.1984 & 706.90 & 283 & 0.5428 & 783.30 & 315 & 0.8950 & 862.55 & 88 \\
\hline 0.2469 & 717.50 & 321 & 0.5953 & 795.08 & 288 & 0.9520 & 875.42 & 41 \\
\hline 0.2976 & 728.70 & 342 & 0.6449 & 806.18 & 269 & 1.0000 & 886.26 & 0 \\
\hline \multicolumn{9}{|c|}{$298.15 \mathrm{~K}$} \\
\hline 0.0000 & 654.87 & 0 & 0.3466 & 729.90 & 349 & 0.6923 & 806.68 & 237 \\
\hline 0.0508 & 665.61 & 115 & 0.3941 & 740.36 & 353 & 0.7451 & 818.46 & 207 \\
\hline 0.0988 & 675.94 & 180 & 0.4489 & 752.48 & 344 & 0.7968 & 829.99 & 176 \\
\hline 0.1497 & 686.89 & 245 & 0.4961 & 762.96 & 330 & 0.8503 & 842.02 & 131 \\
\hline 0.1990 & 697.60 & 287 & 0.5489 & 774.71 & 311 & 0.8967 & 852.42 & 96 \\
\hline 0.2487 & 708.41 & 322 & 0.5956 & 785.13 & 285 & 0.9506 & 864.53 & 50 \\
\hline 0.2994 & 719.51 & 342 & 0.6469 & 796.55 & 261 & 1.0000 & 875.67 & 0 \\
\hline \multicolumn{9}{|c|}{$308.15 \mathrm{~K}$} \\
\hline 0.0000 & 645.56 & 0 & 0.3463 & 719.88 & 365 & 0.6931 & 796.48 & 243 \\
\hline 0.0505 & 656.19 & 107 & 0.3987 & 731.39 & 361 & 0.7436 & 807.65 & 218 \\
\hline 0.0977 & 666.24 & 176 & 0.4440 & 741.31 & 359 & 0.7952 & 819.15 & 184 \\
\hline 0.1477 & 676.90 & 244 & 0.4951 & 752.57 & 346 & 0.8439 & 830.04 & 142 \\
\hline 0.1951 & 687.05 & 299 & 0.5442 & 763.43 & 328 & 0.8919 & 840.79 & 97 \\
\hline 0.2455 & 697.92 & 336 & 0.5954 & 774.78 & 302 & 0.9451 & 852.66 & 56 \\
\hline 0.2994 & 709.63 & 359 & 0.6111 & 778.25 & 294 & 1.0000 & 864.99 & 0 \\
\hline \multicolumn{9}{|c|}{$318.15 \mathrm{~K}$} \\
\hline 0.0000 & 636.37 & 0 & 0.3485 & 710.61 & 370 & 0.6993 & 787.65 & 236 \\
\hline 0.0524 & 647.30 & 114 & 0.3983 & 721.47 & 365 & 0.7510 & 799.10 & 200 \\
\hline 0.1047 & 658.30 & 200 & 0.4483 & 732.38 & 360 & 0.8005 & 810.07 & 164 \\
\hline 0.1513 & 668.19 & 258 & 0.4969 & 743.03 & 348 & 0.8476 & 820.48 & 133 \\
\hline 0.1980 & 678.17 & 301 & 0.5477 & 754.15 & 330 & 0.8970 & 831.46 & 93 \\
\hline 0.2506 & 689.48 & 334 & 0.5981 & 765.27 & 303 & 0.9473 & 842.65 & 47 \\
\hline 0.3012 & 700.36 & 359 & 0.6440 & 775.40 & 275 & 1.0000 & 854.40 & 0 \\
\hline \multicolumn{9}{|c|}{$328.15 \mathrm{~K}$} \\
\hline 0.0000 & 626.94 & 0 & 0.3466 & 700.24 & 364 & 0.6973 & 776.71 & 238 \\
\hline 0.0516 & 637.62 & 113 & 0.3973 & 711.18 & 368 & 0.7456 & 787.32 & 207 \\
\hline 0.1001 & 647.80 & 183 & 0.4455 & 721.63 & 363 & 0.7946 & 798.18 & 158 \\
\hline 0.1494 & 658.21 & 238 & 0.4952 & 732.43 & 351 & 0.8492 & 810.20 & 124 \\
\hline 0.1981 & 668.51 & 289 & 0.5453 & 743.41 & 322 & 0.9011 & 821.68 & 78 \\
\hline 0.2481 & 679.12 & 330 & 0.5957 & 754.41 & 300 & 0.9485 & 832.20 & 32 \\
\hline 0.3018 & 690.60 & 358 & 0.6467 & 765.59 & 273 & 1.0000 & 843.53 & 0 \\
\hline
\end{tabular}




\begin{tabular}{|c|c|c|c|c|c|c|c|c|}
\hline \multicolumn{9}{|c|}{ propyl propanoate (1) + heptane (2) } \\
\hline \multicolumn{9}{|c|}{$288.15 \mathrm{~K}$} \\
\hline 0.0000 & 688.16 & 0 & 0.3967 & 758.93 & 534 & 0.7977 & 840.80 & 306 \\
\hline 0.0498 & 696.42 & 140 & 0.4433 & 767.95 & 531 & 0.8415 & 850.37 & 251 \\
\hline 0.0948 & 704.09 & 233 & 0.5026 & 779.54 & 529 & 0.8839 & 859.77 & 196 \\
\hline 0.1397 & 711.91 & 305 & 0.5507 & 789.15 & 510 & 0.9539 & 875.66 & 77 \\
\hline 0.1899 & 720.76 & 379 & 0.6026 & 799.68 & 481 & 1.0000 & 886.26 & 0 \\
\hline 0.2438 & 730.45 & 441 & 0.6935 & 818.53 & 412 & & & \\
\hline 0.3427 & 748.75 & 503 & 0.7409 & 828.54 & 373 & & & \\
\hline \multicolumn{9}{|c|}{$298.15 \mathrm{~K}$} \\
\hline 0.0000 & 679.62 & 0 & 0.3510 & 740.91 & 553 & 0.6965 & 809.15 & 431 \\
\hline 0.0575 & 689.05 & 166 & 0.3990 & 749.94 & 566 & 0.7458 & 819.49 & 387 \\
\hline 0.1012 & 696.39 & 265 & 0.4452 & 758.74 & 571 & 0.7963 & 830.30 & 321 \\
\hline 0.1472 & 704.32 & 342 & 0.5007 & 769.51 & 564 & 0.8445 & 840.75 & 261 \\
\hline 0.2000 & 713.54 & 422 & 0.5444 & 778.16 & 545 & 0.8891 & 850.50 & 204 \\
\hline 0.2502 & 722.43 & 489 & 0.5989 & 789.12 & 507 & 0.9441 & 862.87 & 108 \\
\hline 0.2983 & 731.20 & 518 & 0.6459 & 798.66 & 481 & 1.0000 & 875.67 & 0 \\
\hline \multicolumn{9}{|c|}{$308.15 \mathrm{~K}$} \\
\hline 0.0000 & 670.96 & 0 & 0.3960 & 740.12 & 545 & 0.7533 & 810.98 & 376 \\
\hline 0.0508 & 679.32 & 119 & 0.4480 & 749.88 & 558 & 0.7932 & 819.41 & 329 \\
\hline 0.0970 & 687.04 & 219 & 0.4934 & 758.58 & 556 & 0.8524 & 832.28 & 228 \\
\hline 0.1564 & 697.11 & 330 & 0.5434 & 768.32 & 542 & 0.8990 & 842.37 & 174 \\
\hline 0.1983 & 704.35 & 397 & 0.6101 & 781.59 & 503 & 0.9555 & 854.94 & 78 \\
\hline 0.2455 & 712.65 & 456 & 0.6391 & 787.37 & 492 & 1.0000 & 864.99 & 0 \\
\hline 0.3355 & 728.87 & 529 & 0.6953 & 798.93 & 431 & & & \\
\hline \multicolumn{9}{|c|}{$318.15 \mathrm{~K}$} \\
\hline 0.0000 & 662.33 & 0 & 0.3501 & 722.19 & 565 & 0.7013 & 790.18 & 438 \\
\hline 0.0496 & 670.29 & 144 & 0.4005 & 731.45 & 581 & 0.7507 & 800.41 & 376 \\
\hline 0.0960 & 677.85 & 268 & 0.4423 & 739.27 & 585 & 0.7900 & 808.64 & 328 \\
\hline 0.1419 & 685.62 & 341 & 0.4961 & 749.49 & 577 & 0.8517 & 821.77 & 246 \\
\hline 0.1963 & 694.91 & 425 & 0.5510 & 760.11 & 555 & 0.8993 & 832.10 & 168 \\
\hline 0.2521 & 704.62 & 494 & 0.5999 & 769.73 & 526 & 0.9407 & 841.27 & 88 \\
\hline 0.2915 & 711.64 & 526 & 0.6456 & 778.85 & 492 & 1.0000 & 854.40 & 0 \\
\hline \multicolumn{9}{|c|}{$328.15 \mathrm{~K}$} \\
\hline 0.0000 & 653.61 & 0 & 0.3446 & 711.71 & 585 & 0.6953 & 778.64 & 478 \\
\hline 0.0515 & 661.80 & 151 & 0.3942 & 720.68 & 609 & 0.7435 & 788.58 & 403 \\
\hline 0.0999 & 669.64 & 267 & 0.4463 & 730.24 & 625 & 0.7921 & 798.59 & 350 \\
\hline 0.1429 & 676.74 & 357 & 0.4924 & 738.92 & 616 & 0.8402 & 808.70 & 283 \\
\hline 0.1847 & 683.77 & 430 & 0.5455 & 749.13 & 583 & 0.8887 & 819.13 & 197 \\
\hline 0.2477 & 694.58 & 512 & 0.6023 & 760.13 & 555 & 0.9520 & 832.93 & 83 \\
\hline 0.2845 & 701.07 & 535 & 0.6488 & 769.36 & 514 & 1.0000 & 843.53 & 0 \\
\hline \multicolumn{9}{|c|}{ propyl propanoate $(1)+$ octane $(2)$} \\
\hline \multicolumn{9}{|c|}{$288.15 \mathrm{~K}$} \\
\hline 0.0000 & 706.62 & 0 & 0.3646 & 760.52 & 619 & 0.8001 & 841.50 & 400 \\
\hline 0.0720 & 716.33 & 206 & 0.4042 & 767.04 & 648 & 0.8479 & 851.66 & 328 \\
\hline 0.1247 & 723.76 & 320 & 0.4592 & 776.44 & 653 & 0.8890 & 860.67 & 252 \\
\hline
\end{tabular}




\begin{tabular}{|c|c|c|c|c|c|c|c|c|}
\hline 0.1691 & 730.19 & 400 & 0.5057 & 784.62 & 643 & 0.9425 & 872.76 & 142 \\
\hline 0.2228 & 738.18 & 489 & 0.6000 & 801.80 & 610 & 1.0000 & 886.26 & 0 \\
\hline 0.2649 & 744.66 & 531 & 0.6509 & 811.43 & 578 & & & \\
\hline 0.3149 & 752.45 & 594 & 0.7047 & 821.97 & 528 & & & \\
\hline \multicolumn{9}{|c|}{$298.15 \mathrm{~K}$} \\
\hline 0.0000 & 698.55 & 0 & 0.3521 & 749.68 & 623 & 0.6983 & 810.99 & 547 \\
\hline 0.0588 & 706.38 & 163 & 0.4086 & 758.89 & 649 & 0.7403 & 819.39 & 480 \\
\hline 0.0984 & 711.81 & 260 & 0.4497 & 765.76 & 664 & 0.8104 & 833.67 & 392 \\
\hline 0.1580 & 720.25 & 373 & 0.5041 & 775.12 & 663 & 0.8536 & 842.81 & 316 \\
\hline 0.2048 & 727.01 & 465 & 0.5531 & 783.83 & 644 & 0.8973 & 852.34 & 229 \\
\hline 0.2710 & 736.99 & 546 & 0.6037 & 793.07 & 618 & 0.9587 & 866.10 & 98 \\
\hline 0.2929 & 740.39 & 566 & 0.6483 & 801.39 & 592 & 1.0000 & 875.67 & 0 \\
\hline \multicolumn{9}{|c|}{$308.15 \mathrm{~K}$} \\
\hline 0.0000 & 690.39 & 0 & 0.3966 & 747.82 & 678 & 0.7451 & 810.38 & 501 \\
\hline 0.0464 & 696.47 & 130 & 0.4537 & 757.25 & 690 & 0.8365 & 828.91 & 369 \\
\hline 0.1008 & 703.73 & 283 & 0.4973 & 764.62 & 692 & 0.8835 & 838.92 & 273 \\
\hline 0.1553 & 711.27 & 404 & 0.5495 & 773.72 & 676 & 0.9302 & 849.14 & 172 \\
\hline 0.2015 & 717.90 & 481 & 0.5929 & 781.51 & 652 & 1.0000 & 864.99 & 0 \\
\hline 0.2519 & 725.28 & 563 & 0.6381 & 789.80 & 622 & & & \\
\hline 0.3500 & 740.39 & 647 & 0.6882 & 799.26 & 573 & & & \\
\hline \multicolumn{9}{|c|}{$318.15 \mathrm{~K}$} \\
\hline 0.0000 & 682.16 & 0 & 0.3498 & 731.29 & 692 & 0.6977 & 791.19 & 601 \\
\hline 0.0615 & 690.08 & 187 & 0.4035 & 739.78 & 719 & 0.7413 & 799.64 & 537 \\
\hline 0.0942 & 694.38 & 281 & 0.4510 & 747.50 & 731 & 0.8100 & 813.28 & 438 \\
\hline 0.1579 & 703.06 & 427 & 0.5062 & 756.76 & 729 & 0.8540 & 822.49 & 335 \\
\hline 0.2036 & 709.51 & 513 & 0.5569 & 765.54 & 708 & 0.8951 & 831.04 & 273 \\
\hline 0.2676 & 718.84 & 602 & 0.6025 & 773.63 & 683 & 0.9609 & 845.49 & 111 \\
\hline 0.2942 & 722.79 & 638 & 0.6502 & 782.33 & 646 & 1.0000 & 854.40 & 0 \\
\hline \multicolumn{9}{|c|}{$328.15 \mathrm{~K}$} \\
\hline 0.0000 & 673.91 & 0 & 0.3550 & 722.99 & 727 & 0.6971 & 781.14 & 618 \\
\hline 0.0719 & 683.11 & 207 & 0.4029 & 730.49 & 747 & 0.7456 & 790.37 & 551 \\
\hline 0.0993 & 686.63 & 297 & 0.4545 & 738.76 & 760 & 0.8122 & 803.46 & 447 \\
\hline 0.1643 & 695.38 & 449 & 0.5061 & 747.22 & 769 & 0.8514 & 811.42 & 370 \\
\hline 0.2115 & 701.89 & 549 & 0.5556 & 755.64 & 750 & 0.8969 & 820.94 & 268 \\
\hline 0.2707 & 710.36 & 644 & 0.6073 & 764.75 & 709 & 0.9606 & 834.72 & 109 \\
\hline 0.2951 & 713.98 & 668 & 0.6537 & 773.08 & 675 & 1.0000 & 843.53 & 0 \\
\hline \multicolumn{9}{|c|}{ propyl propanoate $(1)+$ nonane $(2)$} \\
\hline \multicolumn{9}{|c|}{$288.15 \mathrm{~K}$} \\
\hline 0.0000 & 721.65 & 0 & 0.3476 & 764.85 & 682 & 0.7123 & 824.57 & 601 \\
\hline 0.0357 & 725.57 & 110 & 0.3832 & 770.04 & 693 & 0.7420 & 830.26 & 562 \\
\hline 0.0969 & 732.56 & 276 & 0.4368 & 777.93 & 730 & 0.7962 & 841.00 & 476 \\
\hline 0.1524 & 739.21 & 395 & 0.4948 & 786.96 & 730 & 0.8437 & 850.82 & 391 \\
\hline 0.1976 & 744.81 & 484 & 0.5478 & 795.54 & 723 & 0.8929 & 861.46 & 280 \\
\hline 0.2521 & 751.86 & 565 & 0.5999 & 804.34 & 696 & 0.9513 & 874.73 & 130 \\
\hline 0.3004 & 758.34 & 626 & 0.6548 & 813.96 & 666 & 1.0000 & 886.26 & 0 \\
\hline \multicolumn{9}{|c|}{$298.15 \mathrm{~K}$} \\
\hline
\end{tabular}




\begin{tabular}{|c|c|c|c|c|c|c|c|c|}
\hline 0.0000 & 713.85 & 0 & 0.3411 & 755.42 & 688 & 0.7166 & 815.83 & 609 \\
\hline 0.0401 & 718.21 & 119 & 0.3893 & 762.17 & 735 & 0.7551 & 823.11 & 556 \\
\hline 0.0999 & 724.92 & 286 & 0.4364 & 769.12 & 742 & 0.8029 & 832.37 & 498 \\
\hline 0.1539 & 731.18 & 432 & 0.4913 & 777.45 & 759 & 0.8534 & 842.85 & 377 \\
\hline 0.1969 & 736.51 & 496 & 0.5453 & 786.08 & 736 & 0.9000 & 852.66 & 296 \\
\hline 0.2654 & 745.24 & 601 & 0.6040 & 795.76 & 723 & 0.9476 & 863.38 & 160 \\
\hline 0.2955 & 749.20 & 643 & 0.6487 & 803.51 & 685 & 1.0000 & 875.67 & 0 \\
\hline \multicolumn{9}{|c|}{$308.15 \mathrm{~K}$} \\
\hline 0.0000 & 705.96 & 0 & 0.3457 & 747.34 & 726 & 0.7098 & 804.80 & 654 \\
\hline 0.0496 & 711.26 & 151 & 0.3991 & 754.82 & 753 & 0.7503 & 812.31 & 594 \\
\hline 0.0979 & 716.60 & 288 & 0.4585 & 763.47 & 777 & 0.8121 & 824.31 & 481 \\
\hline 0.1535 & 722.98 & 430 & 0.4951 & 768.96 & 787 & 0.8551 & 833.01 & 394 \\
\hline 0.1988 & 728.39 & 523 & 0.5557 & 778.46 & 776 & 0.8999 & 842.43 & 289 \\
\hline 0.2529 & 735.15 & 608 & 0.5990 & 785.57 & 748 & 0.9519 & 853.85 & 156 \\
\hline 0.2981 & 741.02 & 664 & 0.6620 & 796.30 & 702 & 1.0000 & 864.99 & 0 \\
\hline \multicolumn{9}{|c|}{$318.15 \mathrm{~K}$} \\
\hline 0.0000 & 698.06 & 0 & 0.3375 & 737.63 & 734 & 0.7018 & 793.81 & 672 \\
\hline 0.0444 & 702.66 & 152 & 0.3976 & 745.80 & 786 & 0.7503 & 802.57 & 614 \\
\hline 0.1022 & 708.94 & 320 & 0.4496 & 753.17 & 815 & 0.8029 & 812.61 & 510 \\
\hline 0.1469 & 714.02 & 422 & 0.4941 & 759.75 & 819 & 0.8501 & 821.96 & 410 \\
\hline 0.1719 & 716.92 & 478 & 0.5538 & 768.97 & 805 & 0.9043 & 833.19 & 276 \\
\hline 0.2325 & 724.16 & 604 & 0.5974 & 776.03 & 771 & 0.9535 & 843.92 & 132 \\
\hline 0.2889 & 731.26 & 684 & 0.6430 & 783.58 & 740 & 1.0000 & 854.40 & 0 \\
\hline \multicolumn{9}{|c|}{$328.15 \mathrm{~K}$} \\
\hline 0.0000 & 690.17 & 0 & 0.3410 & 729.28 & 788 & 0.7066 & 784.78 & 711 \\
\hline 0.0493 & 695.17 & 176 & 0.3944 & 736.49 & 816 & 0.7472 & 792.00 & 653 \\
\hline 0.0979 & 700.39 & 309 & 0.4425 & 743.18 & 837 & 0.8030 & 802.38 & 552 \\
\hline 0.1522 & 706.36 & 460 & 0.4955 & 750.83 & 852 & 0.8506 & 811.67 & 442 \\
\hline 0.2078 & 712.79 & 578 & 0.5476 & 758.63 & 852 & 0.8990 & 821.51 & 318 \\
\hline 0.2441 & 717.12 & 646 & 0.5948 & 766.04 & 827 & 0.9482 & 831.98 & 173 \\
\hline 0.2944 & 723.29 & 736 & 0.6431 & 773.91 & 791 & 1.0000 & 843.53 & 0 \\
\hline \multicolumn{9}{|c|}{ butyl propanoate $(1)+$ hexane $(2)$} \\
\hline \multicolumn{9}{|c|}{$288.15 \mathrm{~K}$} \\
\hline 0.0000 & 664.00 & 0 & 0.3471 & 745.00 & 157 & 0.6965 & 820.48 & 91 \\
\hline 0.0454 & 674.90 & 47 & 0.3904 & 754.68 & 158 & 0.7484 & 831.17 & 76 \\
\hline 0.0872 & 684.88 & 82 & 0.4387 & 765.37 & 152 & 0.8023 & 842.10 & 65 \\
\hline 0.1582 & 701.63 & 121 & 0.4929 & 777.27 & 140 & 0.8554 & 852.74 & 53 \\
\hline 0.2052 & 712.59 & 138 & 0.5390 & 787.23 & 131 & 0.8839 & 858.40 & 44 \\
\hline 0.2489 & 722.67 & 148 & 0.5939 & 798.96 & 120 & 0.9413 & 869.63 & 33 \\
\hline 0.2956 & 733.34 & 157 & 0.6465 & 810.06 & 108 & 1.0000 & 881.11 & 0 \\
\hline \multicolumn{9}{|c|}{$298.15 \mathrm{~K}$} \\
\hline 0.0000 & 654.87 & 0 & 0.3471 & 735.76 & 125 & 0.6991 & 811.59 & 68 \\
\hline 0.0452 & 665.79 & 28 & 0.3936 & 746.14 & 122 & 0.7503 & 822.09 & 59 \\
\hline 0.0892 & 676.23 & 61 & 0.4407 & 756.53 & 117 & 0.8071 & 833.57 & 51 \\
\hline 0.1584 & 692.53 & 93 & 0.4959 & 768.59 & 109 & 0.8564 & 843.43 & 40 \\
\hline 0.2065 & 703.71 & 107 & 0.5444 & 779.04 & 99 & 0.8872 & 849.54 & 32 \\
\hline
\end{tabular}




\begin{tabular}{|c|c|c|c|c|c|c|c|c|}
\hline 0.2495 & 713.61 & 118 & 0.5925 & 789.28 & 92 & 0.9451 & 860.87 & 22 \\
\hline 0.2975 & 724.56 & 125 & 0.6438 & 800.08 & 82 & 1.0000 & 871.55 & 0 \\
\hline \multicolumn{9}{|c|}{$308.15 \mathrm{~K}$} \\
\hline 0.0000 & 645.56 & 0 & 0.3471 & 725.89 & 114 & 0.6965 & 800.93 & 29 \\
\hline 0.0521 & 657.85 & 61 & 0.3984 & 737.32 & 102 & 0.7481 & 811.40 & 28 \\
\hline 0.0993 & 669.01 & 86 & 0.4500 & 748.63 & 97 & 0.7985 & 821.60 & 13 \\
\hline 0.1475 & 680.25 & 115 & 0.4980 & 759.10 & 77 & 0.8479 & 831.51 & -7 \\
\hline 0.2022 & 692.98 & 118 & 0.5507 & 770.34 & 75 & 0.9017 & 842.05 & -7 \\
\hline 0.2507 & 704.10 & 123 & 0.5987 & 780.53 & 58 & 0.9479 & 850.98 & -6 \\
\hline 0.3011 & 715.53 & 125 & 0.6467 & 790.61 & 40 & 1.0000 & 861.18 & 0 \\
\hline \multicolumn{9}{|c|}{$318.15 \mathrm{~K}$} \\
\hline 0.0000 & 636.37 & 0 & 0.3474 & 716.68 & 99 & 0.6958 & 791.47 & 20 \\
\hline 0.0453 & 647.15 & 35 & 0.3934 & 726.89 & 94 & 0.7498 & 802.47 & 15 \\
\hline 0.0864 & 656.86 & 59 & 0.4399 & 737.12 & 84 & 0.8043 & 813.50 & 1 \\
\hline 0.1587 & 673.75 & 88 & 0.4946 & 749.03 & 66 & 0.8566 & 823.90 & -5 \\
\hline 0.2049 & 684.43 & 96 & 0.5388 & 758.54 & 54 & 0.8838 & 829.24 & -5 \\
\hline 0.2501 & 694.73 & 105 & 0.5943 & 770.31 & 42 & 0.9608 & 844.19 & -2 \\
\hline 0.2971 & 705.42 & 100 & 0.6539 & 782.79 & 32 & 1.0000 & 851.69 & 0 \\
\hline \multicolumn{9}{|c|}{$328.15 \mathrm{~K}$} \\
\hline 0.0000 & 626.94 & 0 & 0.3478 & 707.05 & 86 & 0.6951 & 781.40 & 11 \\
\hline 0.0448 & 637.54 & 38 & 0.3954 & 717.59 & 78 & 0.7485 & 792.28 & 4 \\
\hline 0.0908 & 648.35 & 62 & 0.4383 & 727.02 & 63 & 0.8002 & 802.69 & -1 \\
\hline 0.1580 & 664.01 & 77 & 0.4910 & 738.47 & 49 & 0.8561 & 813.81 & -5 \\
\hline 0.2028 & 674.32 & 85 & 0.5417 & 749.31 & 41 & 0.8834 & 819.18 & -5 \\
\hline 0.2481 & 684.65 & 91 & 0.5934 & 760.25 & 31 & 0.9420 & 830.56 & -3 \\
\hline 0.2962 & 695.52 & 88 & 0.6446 & 770.97 & 20 & 1.0000 & 841.69 & 0 \\
\hline \multicolumn{9}{|c|}{ butyl propanoate (1) + heptane (2) } \\
\hline \multicolumn{9}{|c|}{$288.15 \mathrm{~K}$} \\
\hline 0.0000 & 688.16 & 0 & 0.4034 & 765.02 & 316 & 0.7459 & 831.56 & 187 \\
\hline 0.0492 & 697.35 & 89 & 0.4406 & 772.20 & 317 & 0.8025 & 842.61 & 145 \\
\hline 0.0998 & 706.92 & 156 & 0.4980 & 783.31 & 308 & 0.8576 & 853.39 & 102 \\
\hline 0.1532 & 717.02 & 218 & 0.5431 & 792.07 & 291 & 0.9087 & 863.43 & 50 \\
\hline 0.1815 & 722.43 & 237 & 0.5939 & 801.93 & 273 & 0.9531 & 872.16 & 5 \\
\hline 0.2523 & 735.96 & 280 & 0.6495 & 812.77 & 244 & 1.0000 & 881.11 & 0 \\
\hline 0.2987 & 744.84 & 303 & 0.6933 & 821.33 & 216 & & & \\
\hline \multicolumn{9}{|c|}{$298.15 \mathrm{~K}$} \\
\hline 0.0000 & 679.62 & 0 & 0.3511 & 745.85 & 343 & 0.7011 & 813.37 & 246 \\
\hline 0.0480 & 688.58 & 79 & 0.4026 & 755.73 & 346 & 0.7501 & 822.86 & 217 \\
\hline 0.1014 & 698.54 & 165 & 0.4500 & 764.84 & 344 & 0.7972 & 832.07 & 174 \\
\hline 0.1543 & 708.45 & 233 & 0.5006 & 774.60 & 332 & 0.8510 & 842.53 & 132 \\
\hline 0.2054 & 718.12 & 276 & 0.5522 & 784.53 & 320 & 0.8972 & 851.50 & 96 \\
\hline 0.2523 & 727.01 & 306 & 0.5984 & 793.45 & 302 & 0.9527 & 862.34 & 42 \\
\hline 0.3002 & 736.16 & 322 & 0.6484 & 803.15 & 276 & 1.0000 & 871.55 & 0 \\
\hline \multicolumn{9}{|c|}{$308.15 \mathrm{~K}$} \\
\hline 0.0000 & 670.96 & 0 & 0.3520 & 736.60 & 375 & 0.6970 & 802.54 & 280 \\
\hline 0.0509 & 680.22 & 116 & 0.3973 & 745.22 & 377 & 0.7512 & 813.03 & 231 \\
\hline
\end{tabular}




\begin{tabular}{|c|c|c|c|c|c|c|c|c|}
\hline 0.0989 & 689.04 & 206 & 0.4500 & 755.24 & 374 & 0.7977 & 822.00 & 195 \\
\hline 0.1514 & 698.85 & 258 & 0.5032 & 765.37 & 368 & 0.8524 & 832.60 & 144 \\
\hline 0.1990 & 707.73 & 306 & 0.5504 & 774.41 & 354 & 0.8993 & 841.65 & 104 \\
\hline 0.2522 & 717.74 & 341 & 0.5977 & 783.47 & 335 & 0.9525 & 851.93 & 57 \\
\hline 0.2993 & 726.60 & 366 & 0.6514 & 793.76 & 309 & 1.0000 & 861.18 & 0 \\
\hline \multicolumn{9}{|c|}{$318.15 \mathrm{~K}$} \\
\hline 0.0000 & 662.33 & 0 & 0.3327 & 724.07 & 357 & 0.7017 & 794.30 & 250 \\
\hline 0.0502 & 671.52 & 90 & 0.4063 & 737.98 & 361 & 0.7405 & 801.71 & 230 \\
\hline 0.0972 & 680.17 & 166 & 0.4507 & 746.42 & 355 & 0.8050 & 814.13 & 178 \\
\hline 0.1493 & 689.80 & 231 & 0.5043 & 756.52 & 360 & 0.8452 & 821.76 & 160 \\
\hline 0.1899 & 697.29 & 282 & 0.5476 & 764.77 & 345 & 0.9038 & 833.13 & 96 \\
\hline 0.2554 & 709.55 & 323 & 0.5994 & 774.70 & 315 & 0.9514 & 842.27 & 55 \\
\hline 0.2972 & 717.35 & 352 & 0.6535 & 785.01 & 290 & 1.0000 & 851.69 & 0 \\
\hline \multicolumn{9}{|c|}{$328.15 \mathrm{~K}$} \\
\hline 0.0000 & 653.61 & 0 & 0.4061 & 728.64 & 368 & 0.7385 & 791.56 & 247 \\
\hline 0.0399 & 660.87 & 72 & 0.4511 & 737.09 & 371 & 0.8531 & 813.43 & 157 \\
\hline 0.0905 & 670.11 & 152 & 0.4965 & 745.69 & 355 & 0.8966 & 821.82 & 107 \\
\hline 0.1605 & 682.98 & 235 & 0.5490 & 755.56 & 351 & 0.9515 & 832.33 & 57 \\
\hline 0.2025 & 690.69 & 282 & 0.5972 & 764.65 & 339 & 1.0000 & 841.69 & 0 \\
\hline 0.2527 & 700.01 & 318 & 0.6497 & 774.64 & 306 & & & \\
\hline 0.3482 & 717.81 & 359 & 0.7154 & 787.12 & 270 & & & \\
\hline \multicolumn{9}{|c|}{ butyl propanoate (1) + octane (2) } \\
\hline \multicolumn{9}{|c|}{$288.15 \mathrm{~K}$} \\
\hline 0.0000 & 706.62 & 0 & 0.3498 & 761.94 & 455 & 0.7003 & 823.38 & 387 \\
\hline 0.0622 & 716.08 & 117 & 0.3950 & 769.49 & 477 & 0.7503 & 832.62 & 349 \\
\hline 0.1080 & 723.06 & 210 & 0.4544 & 779.62 & 482 & 0.7950 & 841.02 & 304 \\
\hline 0.1620 & 731.47 & 294 & 0.4993 & 787.40 & 478 & 0.8434 & 850.24 & 250 \\
\hline 0.2058 & 738.44 & 344 & 0.5515 & 796.52 & 472 & 0.8976 & 860.68 & 184 \\
\hline 0.2536 & 746.12 & 390 & 0.5960 & 804.44 & 452 & 0.9454 & 870.06 & 116 \\
\hline 0.3033 & 754.23 & 430 & 0.6521 & 814.51 & 429 & 1.0000 & 881.11 & 0 \\
\hline \multicolumn{9}{|c|}{$298.15 \mathrm{~K}$} \\
\hline 0.0000 & 698.55 & 0 & 0.3483 & 753.08 & 467 & 0.6977 & 813.85 & 388 \\
\hline 0.0560 & 706.81 & 146 & 0.3937 & 760.64 & 481 & 0.7492 & 823.32 & 340 \\
\hline 0.1090 & 714.87 & 244 & 0.4499 & 770.17 & 481 & 0.7926 & 831.42 & 295 \\
\hline 0.1555 & 722.05 & 313 & 0.4978 & 778.38 & 478 & 0.8437 & 841.03 & 242 \\
\hline 0.2058 & 730.01 & 364 & 0.5512 & 787.65 & 469 & 0.8917 & 850.29 & 169 \\
\hline 0.2545 & 737.76 & 414 & 0.5952 & 795.41 & 449 & 0.9403 & 859.69 & 108 \\
\hline 0.3015 & 745.40 & 444 & 0.6507 & 805.34 & 418 & 1.0000 & 871.55 & 0 \\
\hline \multicolumn{9}{|c|}{$308.15 \mathrm{~K}$} \\
\hline 0.0000 & 690.39 & 0 & 0.3517 & 744.66 & 501 & 0.7015 & 804.85 & 400 \\
\hline 0.0503 & 697.76 & 122 & 0.4009 & 752.76 & 515 & 0.7533 & 814.26 & 353 \\
\hline 0.1012 & 705.41 & 214 & 0.4531 & 761.48 & 519 & 0.7979 & 822.51 & 300 \\
\hline 0.1530 & 713.25 & 307 & 0.4993 & 769.34 & 507 & 0.8477 & 831.76 & 248 \\
\hline 0.2034 & 721.01 & 380 & 0.5535 & 778.63 & 500 & 0.9024 & 842.23 & 159 \\
\hline 0.2532 & 728.84 & 434 & 0.6006 & 786.87 & 473 & 0.9518 & 851.66 & 98 \\
\hline 0.3018 & 736.62 & 464 & 0.6518 & 795.89 & 445 & 1.0000 & 861.18 & 0 \\
\hline
\end{tabular}




\begin{tabular}{|c|c|c|c|c|c|c|c|c|}
\hline \multicolumn{9}{|c|}{$318.15 \mathrm{~K}$} \\
\hline 0.0000 & 682.16 & 0 & 0.3540 & 736.29 & 527 & 0.7032 & 795.96 & 419 \\
\hline 0.0596 & 690.80 & 152 & 0.4004 & 743.83 & 550 & 0.7514 & 804.73 & 362 \\
\hline 0.1106 & 698.35 & 260 & 0.4559 & 753.07 & 549 & 0.7937 & 812.52 & 309 \\
\hline 0.1585 & 705.63 & 330 & 0.5018 & 760.79 & 542 & 0.8453 & 822.04 & 253 \\
\hline 0.2103 & 713.52 & 413 & 0.5519 & 769.34 & 529 & 0.8981 & 831.99 & 177 \\
\hline 0.2556 & 720.58 & 465 & 0.5987 & 777.44 & 504 & 0.9411 & 840.31 & 94 \\
\hline 0.3050 & 728.44 & 496 & 0.6543 & 787.20 & 467 & 1.0000 & 851.69 & 0 \\
\hline \multicolumn{9}{|c|}{$328.15 \mathrm{~K}$} \\
\hline 0.0000 & 673.91 & 0 & 0.3524 & 727.18 & 541 & 0.7018 & 786.23 & 434 \\
\hline 0.0587 & 682.31 & 158 & 0.3972 & 734.40 & 559 & 0.7519 & 795.25 & 376 \\
\hline 0.1050 & 689.04 & 267 & 0.4550 & 743.91 & 557 & 0.7969 & 803.43 & 319 \\
\hline 0.1584 & 697.04 & 352 & 0.5011 & 751.58 & 555 & 0.8444 & 812.15 & 261 \\
\hline 0.2083 & 704.58 & 427 & 0.5532 & 760.39 & 537 & 0.8977 & 822.09 & 185 \\
\hline 0.2551 & 711.87 & 465 & 0.5975 & 767.96 & 518 & 0.9466 & 831.41 & 97 \\
\hline 0.3031 & 719.36 & 509 & 0.6554 & 778.04 & 474 & 1.0000 & 841.69 & 0 \\
\hline \multicolumn{9}{|c|}{ butyl propanoate $(1)+$ nonane $(2)$} \\
\hline \multicolumn{9}{|c|}{$288.15 \mathrm{~K}$} \\
\hline 0.0000 & 721.65 & 0 & 0.3469 & 768.18 & 507 & 0.6953 & 823.89 & 417 \\
\hline 0.0515 & 728.05 & 117 & 0.4008 & 776.16 & 531 & 0.7506 & 833.60 & 371 \\
\hline 0.0905 & 732.99 & 202 & 0.4517 & 783.95 & 531 & 0.7962 & 841.92 & 304 \\
\hline 0.1509 & 740.86 & 309 & 0.5013 & 791.64 & 539 & 0.8500 & 851.86 & 240 \\
\hline 0.1923 & 746.38 & 373 & 0.5541 & 800.11 & 521 & 0.9099 & 863.36 & 138 \\
\hline 0.2532 & 754.77 & 440 & 0.6064 & 808.67 & 501 & 0.9497 & 871.26 & 52 \\
\hline 0.2971 & 760.93 & 486 & 0.6459 & 815.33 & 473 & 1.0000 & 881.11 & 0 \\
\hline \multicolumn{9}{|c|}{$298.15 \mathrm{~K}$} \\
\hline 0.0000 & 713.85 & 0 & 0.3635 & 762.07 & 566 & 0.6896 & 813.76 & 470 \\
\hline 0.0811 & 723.75 & 215 & 0.3976 & 767.13 & 570 & 0.7452 & 823.41 & 420 \\
\hline 0.1210 & 728.89 & 281 & 0.4684 & 777.77 & 589 & 0.8113 & 835.41 & 312 \\
\hline 0.1454 & 732.04 & 326 & 0.4971 & 782.29 & 572 & 0.8363 & 839.92 & 294 \\
\hline 0.2140 & 741.16 & 424 & 0.5503 & 790.66 & 565 & 0.8976 & 851.44 & 195 \\
\hline 0.2600 & 747.39 & 485 & 0.6017 & 798.97 & 545 & 0.9451 & 860.78 & 84 \\
\hline 0.3125 & 754.76 & 532 & 0.6509 & 807.12 & 516 & 1.0000 & 871.55 & 0 \\
\hline \multicolumn{9}{|c|}{$308.15 \mathrm{~K}$} \\
\hline 0.0000 & 705.96 & 0 & 0.3553 & 752.11 & 601 & 0.6983 & 805.59 & 505 \\
\hline 0.0476 & 711.58 & 147 & 0.4037 & 759.12 & 617 & 0.7487 & 814.24 & 453 \\
\hline 0.0978 & 717.72 & 271 & 0.4528 & 766.39 & 628 & 0.8019 & 823.65 & 379 \\
\hline 0.1525 & 724.65 & 371 & 0.4991 & 773.43 & 621 & 0.8507 & 832.55 & 299 \\
\hline 0.1986 & 730.67 & 436 & 0.5525 & 781.69 & 615 & 0.9011 & 841.93 & 211 \\
\hline 0.2516 & 737.68 & 518 & 0.5973 & 788.88 & 586 & 0.9497 & 851.24 & 115 \\
\hline 0.3033 & 744.78 & 569 & 0.6504 & 797.53 & 554 & 1.0000 & 861.18 & 0 \\
\hline \multicolumn{9}{|c|}{$318.15 \mathrm{~K}$} \\
\hline 0.0000 & 698.06 & 0 & 0.3471 & 742.67 & 588 & 0.7006 & 797.01 & 521 \\
\hline 0.0487 & 703.82 & 134 & 0.3968 & 749.71 & 617 & 0.7575 & 806.76 & 447 \\
\hline 0.0998 & 710.00 & 263 & 0.4531 & 757.92 & 638 & 0.8089 & 815.77 & 381 \\
\hline 0.1497 & 716.29 & 349 & 0.4949 & 764.17 & 638 & 0.8604 & 825.16 & 281 \\
\hline
\end{tabular}




\begin{tabular}{ccccccccc}
0.2075 & 723.71 & 449 & 0.5532 & 773.16 & 618 & 0.9017 & 832.73 & 219 \\
0.2593 & 730.57 & 517 & 0.5972 & 780.14 & 590 & 0.9555 & 842.98 & 103 \\
0.2975 & 735.74 & 559 & 0.6474 & 788.21 & 566 & 1.0000 & 851.69 & 0 \\
\hline \multicolumn{7}{c}{$328.15 \mathrm{~K}$} \\
\hline 0.0000 & 690.17 & 0 & 0.3525 & 734.84 & 616 & 0.6892 & 785.83 & 549 \\
0.0480 & 695.73 & 145 & 0.3946 & 740.73 & 643 & 0.7430 & 794.85 & 487 \\
0.0948 & 701.36 & 251 & 0.4526 & 749.10 & 654 & 0.8009 & 804.85 & 402 \\
0.1492 & 708.03 & 371 & 0.4942 & 755.21 & 661 & 0.8510 & 813.73 & 320 \\
0.1940 & 713.72 & 442 & 0.5527 & 764.08 & 646 & 0.9057 & 823.71 & 219 \\
0.2617 & 722.51 & 538 & 0.6027 & 771.86 & 623 & 0.9543 & 832.84 & 117 \\
0.3060 & 728.47 & 579 & 0.6507 & 779.54 & 584 & 1.0000 & 841.69 & 0 \\
\hline
\end{tabular}

Table S7. Coefficients of Eq 1 at $298.15 \mathrm{~K}$ and Root Squared Mean Error, $s(\rho)$

\begin{tabular}{|llcccc|}
\hline System & & $\theta_{0}$ & $\theta_{1}$ & $\theta_{2}$ & $s(\rho) / \mathrm{kg} \cdot \mathrm{m}^{-3}$ \\
\hline propyl propanoate(1) & + hexane(2) & -9.608 & 10.592 & -5.679 & 0.017 \\
& + heptane(2) & -34.002 & 4.913 & -5.672 & 0.026 \\
& + octane(2) & -46.215 & -6.890 & -4.403 & 0.022 \\
& $+\operatorname{nonane}(2)$ & -56.265 & -7.957 & -13.929 & 0.045 \\
butyl propanoate(1) & $+\operatorname{hexane}(2)$ & 24.777 & 1.802 & -2.516 & 0.018 \\
& + heptane(2) & -6.553 & 4.795 & -1.410 & 0.016 \\
& + octane(2) & -26.554 & 6.293 & -7.023 & 0.019 \\
& + nonane(2) & -37.503 & -4.124 & -1.979 & 0.042 \\
\hline
\end{tabular}

$s(\rho)=\left[\sum_{\mathrm{i}=1}^{\mathrm{m}_{\mathrm{\rho}}}\left(\rho_{\mathrm{i}, \text { xp }}-\rho_{\mathrm{i}, \mathrm{cal}}\right)^{2} / m_{\mathrm{\rho}}\right]^{0.5}$ 
Table S8. Excess molar enthalpies $h^{\mathrm{E}}$ for the binaries an alkyl propanoate (1)+hexane or +octane (2) at $T=298.15$ and $318.15 \mathrm{~K}$ and atmospheric pressure $(p \approx 98.9 \mathrm{kPa})$.

\begin{tabular}{|c|c|c|c|c|c|c|c|}
\hline$x_{1}$ & $\frac{h^{\mathrm{E}}}{\mathrm{J} \cdot \mathrm{mol}^{-1}}$ & $x_{1}$ & $\frac{h^{\mathrm{E}}}{\mathrm{J} \cdot \mathrm{mol}^{-1}}$ & $x_{1}$ & $\frac{h^{\mathrm{E}}}{\mathrm{J} \cdot \mathrm{mol}^{-1}}$ & $x_{1}$ & $\frac{h^{\mathrm{E}}}{\mathrm{J} \cdot \mathrm{mol}^{-}}$ \\
\hline \multicolumn{8}{|c|}{ propyl propanoate (1) + hexane (2) } \\
\hline \multicolumn{8}{|c|}{$318.15 \mathrm{~K}$} \\
\hline 0.0337 & 103 & 0.2918 & 698 & 0.5440 & 804 & 0.8673 & 336 \\
\hline 0.0642 & 203 & 0.3735 & 799 & 0.6175 & 742 & 0.9439 & 168 \\
\hline 0.1307 & 390 & 0.4336 & 830 & 0.6971 & 641 & & \\
\hline 0.2280 & 593 & 0.4796 & 833 & 0.7847 & 504 & & \\
\hline \multicolumn{8}{|c|}{ propyl propanoate (1) + octane (2) } \\
\hline \multicolumn{8}{|c|}{$318.15 \mathrm{~K}$} \\
\hline 0.0617 & 247 & 0.4541 & 997 & 0.6365 & 900 & 0.9126 & 300 \\
\hline 0.1306 & 447 & 0.4988 & 992 & 0.6909 & 826 & 0.9554 & 159 \\
\hline 0.2068 & 631 & 0.5239 & 990 & 0.7453 & 729 & & \\
\hline 0.2907 & 815 & 0.5414 & 981 & 0.8043 & 604 & & \\
\hline 0.3761 & 931 & 0.5887 & 947 & 0.8620 & 457 & & \\
\hline \multicolumn{8}{|c|}{ butyl propanoate $(1)+$ hexane $(2)$} \\
\hline \multicolumn{8}{|c|}{$298.15 \mathrm{~K}$} \\
\hline 0.0493 & 112 & 0.3484 & 659 & 0.5311 & 671 & 0.8609 & 269 \\
\hline 0.1062 & 248 & 0.4053 & 687 & 0.5884 & 633 & 0.9338 & 150 \\
\hline 0.1664 & 375 & 0.4353 & 699 & 0.6492 & 576 & & \\
\hline 0.2280 & 493 & 0.4552 & 694 & 0.7176 & 498 & & \\
\hline 0.2919 & 594 & 0.4807 & 692 & 0.7881 & 394 & & \\
\hline \multicolumn{8}{|c|}{$318.15 \mathrm{~K}$} \\
\hline 0.0602 & 144 & 0.3352 & 653 & 0.4913 & 687 & 0.7695 & 436 \\
\hline 0.1223 & 286 & 0.4007 & 693 & 0.5232 & 677 & 0.8614 & 278 \\
\hline 0.1799 & 426 & 0.4166 & 695 & 0.5983 & 630 & 0.9398 & 136 \\
\hline 0.2636 & 585 & 0.4586 & 695 & 0.6790 & 553 & & \\
\hline \multicolumn{8}{|c|}{ butyl propanoate (1) + octane (2) } \\
\hline \multicolumn{8}{|c|}{$298.15 \mathrm{~K}$} \\
\hline 0.0616 & 210 & 0.4110 & 805 & 0.5372 & 819 & 0.8256 & 437 \\
\hline 0.1327 & 388 & 0.4500 & 823 & 0.5904 & 792 & 0.8902 & 293 \\
\hline 0.2071 & 541 & 0.4688 & 824 & 0.6436 & 745 & 0.9451 & 160 \\
\hline 0.2773 & 656 & 0.4907 & 829 & 0.6994 & 669 & & \\
\hline 0.3474 & 758 & 0.5187 & 827 & 0.7618 & 561 & & \\
\hline \multicolumn{8}{|c|}{$318.15 \mathrm{~K}$} \\
\hline 0.0644 & 218 & 0.4137 & 838 & 0.6113 & 790 & 0.7293 & 637 \\
\hline 0.1472 & 429 & 0.4886 & 855 & 0.6479 & 751 & 0.8106 & 482 \\
\hline 0.2384 & 616 & 0.5555 & 842 & 0.7293 & 637 & 0.8883 & 304 \\
\hline 0.3295 & 757 & 0.5739 & 824 & 0.8106 & 482 & 0.9593 & 123 \\
\hline
\end{tabular}


Table S9. Experimental $(T, x, y)$ iso- $p$ VLE data obtained at $101.32 \mathrm{kPa}$, and calculated properties for the binaries an ester(1)+an alkane(2).

\begin{tabular}{|c|c|c|c|c|c|c|c|c|c|c|c|}
\hline$T / \mathrm{K}$ & $x_{1}$ & $y_{1}$ & $\gamma_{1}$ & $\gamma_{2}$ & $g^{\mathrm{E} / R T}$ & $T / \mathrm{K}$ & $x_{1}$ & $y_{1}$ & $\gamma_{1}$ & $\gamma_{2}$ & $g^{\mathrm{E} / R T}$ \\
\hline \multicolumn{12}{|c|}{ propyl propanoate(1)+hexane $(2)$} \\
\hline 341.82 & 0.000 & 0.000 & - & 1.000 & 0.000 & 353.45 & & & & & 0.145 \\
\hline 342.08 & 0.009 & 0.003 & 2.047 & 0.999 & 0.005 & 354.09 & & & & & .146 \\
\hline 342.24 & 0.016 & 0.005 & 1.906 & 0.999 & 0.009 & 355.20 & & & 1.169 & 1.150 & .148 \\
\hline 42.41 & 0.024 & 0.007 & 1.766 & 1.000 & 0.014 & 355.86 & & & & & .144 \\
\hline 342.82 & & 0.013 & 1.843 & 1.000 & 0.026 & 356.37 & & & & & .147 \\
\hline 343.18 & & 0.017 & & 1.002 & 0.034 & 358.56 & & & & 1.180 & 140 \\
\hline 343.55 & & 0.022 & & 1.005 & 0.044 & 359.40 & & & & & 137 \\
\hline 344.06 & 0.09 & 0.029 & 1.7 & 1.004 & 0.056 & 361.06 & & & 95 & 1.218 & 130 \\
\hline 344 & & 0.035 & 1.688 & 1.007 & 0.066 & 362.18 & & & & 1.229 & 123 \\
\hline 345.00 & & 0.041 & & 1.010 & 0.076 & 363.20 & & & & 53 & 119 \\
\hline 345.49 & & 0.047 & 1. & 1.014 & 0.086 & 366.36 & & & 1.067 & 1.270 & 113 \\
\hline & & & & 1.020 & 0.093 & 372.16 & & & & & 094 \\
\hline 46.49 & 0.1 & 0.059 & & 1.022 & 0.102 & 374.93 & & & 36 & & 0.078 \\
\hline & & & & 1.038 & & & & & & & 069 \\
\hline 3 & & 0.079 & & 1.040 & 0.1 & 380.7 & & & & 1.406 & 0.054 \\
\hline & & 0.08 & & 1.057 & & & & & & & 027 \\
\hline 3 & & 0.096 & & 1.060 & 0.136 & 389 & $0 . S$ & & 1.0 & 1.4 & 0.021 \\
\hline .08 & & 0.100 & & 1.063 & 0 & 391.08 & & & 07 & 1.419 & 0.016 \\
\hline & & & & 1.072 & & & & & & & 010 \\
\hline 350.40 & & 0.103 & 1.2 & 1.082 & 0.138 & 393.08 & & & 1.003 & 1.367 & 0.007 \\
\hline & & & & 1.089 & & 395.32 & & & 1.000 & - & 000 \\
\hline & & & & 1.098 & 0.144 & & & & & & \\
\hline \multicolumn{12}{|c|}{ propyl propanoate(1)+heptane(2) } \\
\hline & & & - & & & & & & & & \\
\hline & & 0. & & 0.994 & & 378.93 & & & & & .115 \\
\hline & 0.02 & 0.021 & 1.582 & 0.995 & 0.007 & 379.17 & 0.5 & 0.3 & 1.087 & 1.172 & 115 \\
\hline 71.96 & & & & 0.994 & & .46 & & & & & .113 \\
\hline 372.03 & 0.044 & 0.034 & 1.564 & 0.996 & 0.015 & 379.79 & 0.607 & 0.413 & 1.073 & 1.194 & 0.112 \\
\hline & & & & 0.997 & & 380.48 & & & & & 0.110 \\
\hline 372.34 & 0.082 & 0.062 & 1.515 & 0.998 & 0.032 & 380.81 & 0.653 & 0.453 & 1.060 & 1.227 & 0.109 \\
\hline & & & & 0.999 & & & & & & & 0.104 \\
\hline 372.56 & 0.10 & 0.080 & 1.473 & 1.001 & 0.0 & & 0.691 & 0.4 & 53 & 1.240 & 0.103 \\
\hline & & & & 1.0 & & & & & & & 0.099 \\
\hline & & 0.105 & & 1.006 & & 382. & 0.719 & 0.5 & 1.046 & 1.2 & 0.096 \\
\hline 373.05 & 0.162 & 0.116 & 1.401 & 1.010 & 0.063 & 383.31 & 0.736 & 0.539 & 1.037 & 1.274 & 0.091 \\
\hline & & 0.130 & & 1.014 & & 383.81 & & & 37 & 1.2 & 0.088 \\
\hline 373.43 & 0.201 & 0.141 & 1.356 & 1.019 & 0.076 & 384.40 & 0.766 & 0.577 & 1.032 & 1.283 & 0.083 \\
\hline & & & & 1.024 & & & & & 29 & 1.285 & 0.075 \\
\hline 373.80 & 0.234 & 0.163 & 1.330 & 1.025 & 0.086 & 386.26 & 0.815 & 0.642 & 1.021 & 1.311 & 0.067 \\
\hline & 0.253 & 0.174 & 1.305 & 1.032 & 0.091 & 386.86 & 0.828 & 0.664 & 1.021 & 1.303 & 0.063 \\
\hline 374.15 & 0.269 & 0.184 & 1.291 & 1.037 & 0.096 & 386.73 & 0.829 & 0.661 & 1.019 & 1.327 & 0.064 \\
\hline 374.37 & 0.286 & 0.195 & 1.278 & 1.042 & 0.099 & 387.55 & 0.843 & 0.686 & 1.016 & 1.312 & 0.056 \\
\hline
\end{tabular}




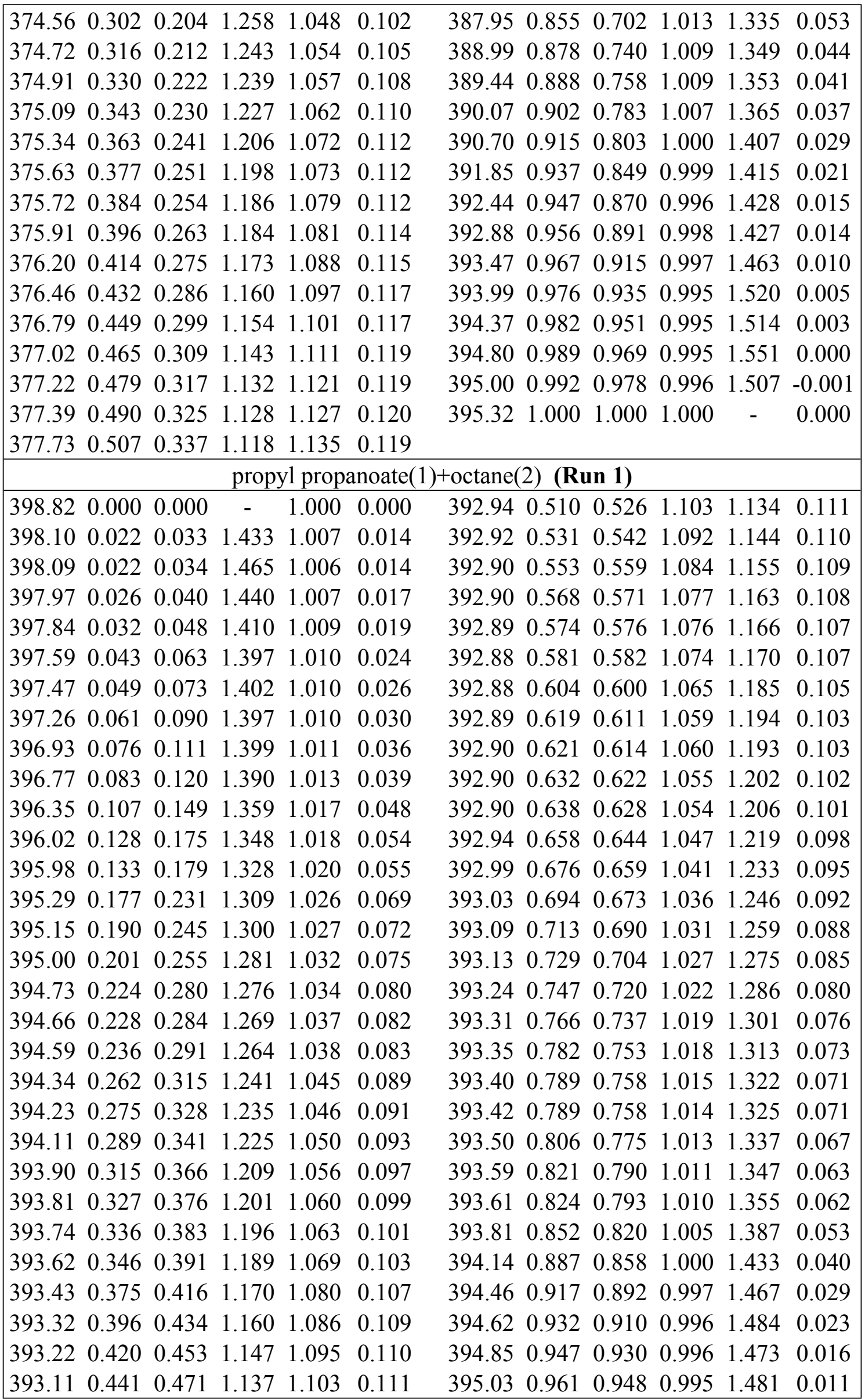




\begin{tabular}{|c|c|c|c|c|c|c|c|c|c|c|}
\hline & 0.40 & 0.49 & & & & 95.200 .5 & & & & \\
\hline & 0.475 & 0.499 & 1.121 & 1.116 & 0.112 & 95.320 .977 & 0.968 & 0.992 & 1.517 & 0.002 \\
\hline 97 & 0.489 & 0.509 & 1.113 & 1.123 & & 395.321 .000 & 1.000 & 1.000 & - & 0.000 \\
\hline \multicolumn{11}{|c|}{ propyl propanoate $(1)+$ nonane $(2)$} \\
\hline 423.90 & 0.000 & 0.000 & - & 1.000 & 0.000 & 402.460 .507 & 0.689 & 1.124 & 1.117 & 0.114 \\
\hline 422.22 & 0.025 & 0.071 & 1.459 & 0.988 & -0.003 & $402.20 \quad 0.521$ & 0.695 & 1.111 & 1.136 & 0.116 \\
\hline & 0.032 & 0.090 & 1.463 & 0.988 & 0.001 & 401.620 .550 & 0.713 & 1.096 & 1.157 & 0.116 \\
\hline 420.94 & 0.043 & 0.116 & 1.426 & 50.989 & 0.005 & 401.190 .577 & 0.727 & 1.077 & 1.186 & 0.115 \\
\hline 420.33 & 0.053 & 0.138 & 1.395 & 0.990 & 0.008 & 400.930 .590 & 0.736 & 1.074 & 1.192 & 0.114 \\
\hline 419.56 & .0 & .167 & 1.4 & 0.988 & 0.0 & .510 .616 & 0.755 & 1.067 & 1.196 & 0.109 \\
\hline 4 & 0.076 & 0.193 & 1.4 & 0.987 & 0.014 & 170.633 & 0.766 & 1.063 & 1.207 & 0.108 \\
\hline 18 & 0.086 & 0.215 & 1.407 & 0.987 & 0.0 & .660 .660 & 0.781 & 1.053 & 1.238 & 0.107 \\
\hline 17.38 & 0.10 & 0.242 & 1.387 & 0.988 & 0.0 & 420.676 & 0.792 & 1.050 & 1.243 & 0.103 \\
\hline 416.69 & 0.113 & 0.265 & 1.366 & 50.990 & 0.026 & 399.090 .695 & 0.801 & 1.042 & 1.276 & 0.103 \\
\hline 415.85 & 017 & 0.292 & 1.3 & 0.993 & 0.0 & $\begin{array}{ll}77 & 0.7\end{array}$ & 15 & 1.028 & & 0.096 \\
\hline 415.02 & 0.144 & 0.319 & 1.342 & 0.993 & 0.037 & $.48 \quad 0.742$ & 0.830 & 1.028 & 1.313 & 0.090 \\
\hline & 16 & 0.348 & 1.3 & 0.996 & & 150.7 & 0.8 & & & 0.086 \\
\hline 413.36 & 0.179 & 0.372 & 1.310 & 0.998 & 0.0 & $\begin{array}{ll}85 & 0.789\end{array}$ & 0.859 & 1.017 & 1.357 & 0.078 \\
\hline 412 & 0.19 & 0.398 & 1.293 & 1.003 & & 490.8 & 0.8 & 1.0 & 1.372 & 0.074 \\
\hline 411.9 & 0.212 & 0.417 & 1.2 & 1.004 & 0.056 & 280.8 & 0.877 & 1.017 & 1.396 & 0.075 \\
\hline 17 & 0.229 & 0.440 & 1.276 & 51.006 & 0.0 & .220 .835 & 0.885 & 1.007 & 1.443 & 0.066 \\
\hline & 0 & 0.457 & 1.256 & 61.012 & & 960.8 & 0.899 & 1.007 & & .060 \\
\hline 409 & 0.263 & 0.477 & 1.241 & 1.016 & 0.0 & 396.680 .874 & 0.913 & 1.007 & 1.453 & 0.054 \\
\hline 409 & $0.2^{-}$ & 0.491 & 1.232 & 1.020 & & $48 \quad 0.8$ & 0.925 & 1.003 & 499 & 0.046 \\
\hline 4 & 0.30 & 0.518 & 1.2 & 71.029 & & 270.9 & 0.935 & 0.998 & 1.611 & 0.039 \\
\hline 408.09 & 0.314 & 0.530 & 1.210 & 1.033 & 0.0 & 120.9 & 0.947 & 0.998 & 1.599 & 0.032 \\
\hline & & 0 & 1.2 & 1.044 & & $\begin{array}{ll}38 & 0.9\end{array}$ & 0.9 & 0.5 & 1.669 & 0.025 \\
\hline 406 & 0.365 & 0.576 & 1.182 & 1.058 & 0.0 & $\begin{array}{ll}70 & 0.9\end{array}$ & 0.965 & 1.002 & 1.651 & 0.025 \\
\hline & 0.3 & & 1.1 & 51.0 & & $\begin{array}{ll}80 & 0.9\end{array}$ & 0.9 & 0.5 & & 0.018 \\
\hline & 035 & 0.605 & 1.168 & 1.070 & 0.1 & 530.5 & 0.984 & 0.999 & 1.746 & 0.010 \\
\hline & 0.43 & 0.633 & & & & 80 & 0.991 & 0.999 & 1.788 & 0.005 \\
\hline & 0.4 & 0.644 & 1.1 & 1.092 & & 520.5 & 0.993 & 0.996 & 1.910 & 0.002 \\
\hline & 0.460 & 0.653 & 1.139 & 1.099 & 0.111 & $423.90 \quad 1.000$ & 1.000 & 1.000 & - & 0.000 \\
\hline & & 0.676 & 1.124 & +1.115 & 0.113 & & & & & \\
\hline \multicolumn{11}{|c|}{ butyl propanoate(1)+hexane(2) } \\
\hline & & & - & 1.0 & & 870. & & & & 0.151 \\
\hline & 0.01 & 0.001 & 1.215 & 1.002 & 0.0 & 358.390 .510 & 0.081 & 1.148 & 1.178 & 0.151 \\
\hline 342.31 & 0.015 & 0.002 & 1.928 & 0.999 & 0.009 & 359.250 .518 & 0.086 & 1.159 & 1.165 & 0.150 \\
\hline & 0.022 & 0.003 & 1.958 & 31.000 & & 360.290 .544 & 0.091 & 1.120 & 1.192 & 0.142 \\
\hline 342.77 & 0.034 & 0.004 & 1.666 & 51.003 & 0.020 & 361.420 .565 & 0.099 & 1.122 & 1.202 & 0.145 \\
\hline & 0.068 & 0.008 & 1.608 & 1.012 & 0.0 & $\begin{array}{ll}31 & 0.591\end{array}$ & 0.111 & 1.116 & 1.202 & 0.140 \\
\hline & 0.072 & 0.009 & 1.697 & 71.011 & 0.048 & 365.010 .615 & 0.122 & 1.104 & 1.208 & 0.134 \\
\hline & 0.083 & 0.010 & 1.620 & 1.015 & 0.0 & 369.560 .679 & 0.158 & 1.089 & 1.242 & 0.128 \\
\hline & 0.091 & 0.011 & 1.611 & 1.017 & 0.05 & 371.490 .701 & 0.174 & 1.082 & 1.248 & 0.122 \\
\hline & 0.101 & 0.012 & 1.568 & 31.021 & 0.064 & 372.670 .721 & 0.184 & 1.065 & 1.285 & 0.116 \\
\hline 344.61 & 0.111 & 0.013 & 1.525 & 1.023 & 0.067 & $\begin{array}{lll}373.67 & 0.733 \\
\end{array}$ & 0.193 & 1.060 & 1.297 & 0.112 \\
\hline
\end{tabular}




\begin{tabular}{|c|c|c|c|c|c|c|c|c|c|c|}
\hline & & & 1.566 & 1.025 & & $375.30 \quad 0.745$ & 0.208 & & & \\
\hline & 0.135 & 0.016 & 1.498 & 1.028 & 0.078 & $\begin{array}{lll}379.06 & 0.781\end{array}$ & 0.246 & 1.047 & 1.304 & 0.094 \\
\hline 345.52 & 0.140 & 0.017 & 1.518 & 1.025 & 0.080 & 380.360 .795 & 0.260 & 1.039 & 1.328 & 0.089 \\
\hline & 0.150 & 0.018 & 1.480 & 1.027 & 0.082 & $\begin{array}{lll}383.71 & 0.819\end{array}$ & 0.296 & 1.025 & 1.328 & 0.071 \\
\hline 346.20 & 0.161 & 0.020 & 1.507 & 1.027 & 0.089 & $\begin{array}{lll}387.31 & 0.848\end{array}$ & 0.345 & 1.023 & 1.361 & 0.066 \\
\hline 46.59 & 0.177 & 0.021 & 1.414 & 1.035 & 0.089 & 390.310 .868 & 0.387 & 1.017 & 1.377 & 0.057 \\
\hline 347.09 & 0.191 & 0.023 & 1.404 & 1.035 & 0.093 & 392.040 .878 & 0.412 & 1.013 & 1.378 & 0.051 \\
\hline 37.08 & 0.19 & 0.024 & 1.421 & 1.042 & 0.103 & 393.160 .886 & 0.431 & 1.014 & 1.395 & 0.050 \\
\hline 47.46 & 0.205 & 0.025 & 1.399 & 1.040 & 0.100 & $\begin{array}{ll}395.91 & 0.899\end{array}$ & 0.475 & 1.011 & 1.374 & 0.042 \\
\hline 347.94 & 0.224 & 0.027 & 1.354 & 1.049 & 0.105 & $\begin{array}{lll}399.86 & 0.919\end{array}$ & 0.549 & 1.013 & 1.362 & 0.037 \\
\hline 48.42 & 0.241 & 0.029 & 1.323 & 1.056 & 0.109 & $400.98 \quad 0.925$ & 0.566 & 1.004 & 1.385 & 0.028 \\
\hline 348.89 & 0.257 & 0.032 & 1.342 & 1.061 & 0.120 & $402.84 \quad 0.934$ & 0.608 & 1.011 & 1.372 & 0.031 \\
\hline 350.08 & 0.299 & 0.038 & 1.300 & 1.081 & 0.133 & 404.770 .943 & 0.646 & 1.005 & 1.383 & 0.023 \\
\hline 351.26 & 0.338 & 0.044 & 1.266 & 1.101 & 0.143 & 409.650 .965 & 0.757 & 1.002 & 1.413 & 0.014 \\
\hline 52.24 & 0.359 & 0.048 & 1.247 & 1.102 & 0.141 & $\begin{array}{lll}415.27 & 0.988\end{array}$ & 0.908 & 1.006 & 1.413 & 0.010 \\
\hline 353.26 & 392 & 0.054 & 1.231 & 1.122 & 0.151 & 417.530 .996 & 0.970 & 1.003 & 1.330 & 0.004 \\
\hline 354.18 & 0.414 & 0.058 & 1.204 & 1.131 & 0.149 & 418.701 .000 & 1.000 & 1.000 & - & 0.000 \\
\hline 33 & 0.452 & 0.069 & 1.200 & 1.127 & 0.148 & & & & & \\
\hline \multicolumn{11}{|c|}{ butyl propanoate(1)+heptane(2) } \\
\hline 371.38 & 0.000 & 0.000 & - & 1.000 & 0.000 & 381.050 .400 & 0.149 & 1.154 & 1.096 & 0.113 \\
\hline 371.66 & 0.007 & 0.002 & 1.236 & 1.000 & 0.002 & 381.480 .411 & 0.155 & 1.152 & 1.096 & 0.112 \\
\hline 372.26 & 0.035 & 0.013 & 1.572 & 1.001 & 0.017 & 381.820 .427 & 0.160 & 1.131 & 1.111 & 0.113 \\
\hline 372.47 & 0.044 & 0.016 & 1.527 & 1.002 & 0.020 & 382.320 .442 & 0.167 & 1.121 & 1.116 & 0.112 \\
\hline & 0.05 & 0.0 & & 1.003 & 0.025 & 040. & 79 & & 1.124 & 0.114 \\
\hline 372.93 & 0.065 & 0.024 & 1.525 & 1.003 & 0.030 & 384.130 .499 & 0.196 & 1.097 & 1.146 & 0.114 \\
\hline 373.24 & 0.080 & 0.029 & 1.480 & 1.006 & 0.036 & $\begin{array}{lll}384.70 & 0.509\end{array}$ & 0.204 & 1.098 & 1.141 & 0.112 \\
\hline 373.52 & 0.094 & 0.034 & 1.462 & 1.008 & 0.043 & 386.660 .562 & 0.235 & 1.073 & 1.170 & 0.108 \\
\hline 373.96 & 0.112 & 0.041 & 1.456 & 1.009 & 0.050 & 387.240 .576 & 0.247 & 1.080 & 1.172 & 0.112 \\
\hline 374.21 & 0.124 & 0.045 & 1.430 & 1.011 & 0.054 & 388.990 .624 & 0.277 & 1.056 & 1.215 & 0.107 \\
\hline 374.56 & 0.138 & 0.051 & 1.438 & 1.012 & 0.060 & 391.150 .663 & 0.312 & 1.044 & 1.224 & 0.097 \\
\hline 374.88 & 0.152 & 0.057 & 1.443 & 1.013 & 0.067 & 391.980 .683 & 0.329 & 1.041 & 1.244 & 0.097 \\
\hline 375.23 & 0.169 & 0.062 & 1.394 & 1.018 & 0.071 & 394.300 .721 & 0.372 & 1.037 & 1.251 & 0.089 \\
\hline 375.59 & 0.179 & 0.066 & 1.383 & 1.017 & 0.071 & 150.736 & 0.391 & 1.040 & 1.257 & 0.089 \\
\hline 375.90 & 0.196 & 0.072 & 1.363 & 1.023 & 0.079 & $\begin{array}{ll}396.13 & 0.747\end{array}$ & 0.409 & 1.040 & 1.244 & 0.084 \\
\hline 376.36 & 0.212 & 0.078 & 1.343 & 1.024 & 0.081 & $\begin{array}{lll}398.14 & 0.777\end{array}$ & 0.446 & 1.025 & 1.262 & 0.071 \\
\hline 376.67 & 0.231 & 0.084 & 1.313 & 1.034 & 0.088 & 398.850 .790 & 0.465 & 1.029 & 1.273 & 0.073 \\
\hline 377.63 & 0.272 & 0.098 & 1.257 & 1.048 & 0.096 & $402.06 \quad 0.832$ & 0.533 & 1.017 & 1.292 & 0.057 \\
\hline 378.21 & 0.297 & 0.108 & 1.243 & 1.057 & 0.104 & 403.740 .851 & 0.573 & 1.018 & 1.283 & 0.052 \\
\hline 378.36 & 0.304 & 0.110 & 1.231 & 1.061 & 0.104 & 404.780 .864 & 0.599 & 1.017 & 1.290 & 0.049 \\
\hline 378.63 & 0.313 & 0.114 & 1.227 & 1.062 & 0.106 & $405.03 \quad 0.865$ & 0.602 & 1.013 & 1.282 & 0.045 \\
\hline 378.95 & 0.323 & 0.118 & 1.217 & 1.064 & 0.105 & 407.790 .898 & 0.675 & 1.011 & 1.305 & 0.037 \\
\hline 379.19 & 0.334 & 0.122 & 1.207 & 1.070 & 0.108 & $409.42 \quad 0.915$ & 0.720 & 1.011 & 1.303 & 0.033 \\
\hline 379.52 & 0.348 & 0.126 & 1.183 & 1.078 & 0.108 & 412.470 .946 & 0.809 & 1.010 & 1.312 & 0.024 \\
\hline 379.84 & 0.357 & 0.131 & 1.185 & 1.078 & 0.109 & $415.50 \quad 0.973$ & 0.897 & 1.003 & 1.329 & 0.010 \\
\hline 380.20 & 0.372 & 0.136 & 1.167 & 1.087 & 0.110 & $\begin{array}{lll}417.07 & 0.987\end{array}$ & 0.951 & 1.005 & 1.272 & 0.008 \\
\hline 380.66 & 0.386 & 0.144 & 1.172 & 1.089 & 0.113 & $418.70 \quad 1.000$ & 1.000 & 1.000 & - & 0.000 \\
\hline
\end{tabular}




\begin{tabular}{|c|c|c|c|c|c|c|c|c|c|c|c|}
\hline \multicolumn{12}{|c|}{ butyl propanoate(1)+octane(2) } \\
\hline 398.82 & 0.000 & 0.000 & - & 1.000 & 0.000 & & 0.5 & 0 . & 1.116 & 1.093 & 0 \\
\hline & & 0.025 & & & & & & & & & \\
\hline & & 0.032 & & 1.000 & & & & & & 1.096 & \\
\hline & 0.049 & 0.039 & 1.377 & 0.999 & & & & & & & 99 \\
\hline & 0.070 & & & 1.001 & & & & & & & \\
\hline & & 0.066 & 1.365 & 1.000 & & & & & & & \\
\hline & & & 1.352 & 1.002 & & & & & & & \\
\hline & $0.0 S$ & 0.075 & 1.33 & 1.003 & & & 0.6 & & 1.079 & & \\
\hline & & & & 1.0 & & & & & & & \\
\hline & & 0.102 & 1.32 & 1.006 & 0.0 & & 0.6 & & 67 & 35 & \\
\hline & & & 1.30 & 1.009 & & & 0.7 & & & & 83 \\
\hline & & & & 1.0 & & & & & & & \\
\hline 29 & 0.1 & & 1.28 & 1.014 & & & 0.7 & & & & 81 \\
\hline & & & & 1.0 & & & & & & & \\
\hline & & & 1.2 & 1.02 & & & 0.7 & & & & \\
\hline & & & & 1.0 & & & & & & & \\
\hline 5 & 0.2 & 0.19 & 1.2 & 1.02 & & & 0.8 & & & & \\
\hline & 0 & & & 1.0 & & & & & & & \\
\hline & & & & & & & & & & & \\
\hline & & 0.2 & & 1.042 & & & 0.8 & & & & \\
\hline & 0.3 & & & 1.0 & & & & & & & \\
\hline & 0.3 & 0.247 & 1.2 & 1.03 & & & 0.9 & & 1 . & & \\
\hline & 0.34 & 0.25 & 1.1 & 1.0 & & & & & 20 & & \\
\hline & & 0.273 & & 1.0 & & & & & & & \\
\hline & 0.3 & 0.28 & 1.16 & 1.0 & & & & & & & 27 \\
\hline & & & & 1.0 & & & & & & & \\
\hline & 0.4 & 0.3 & 1.16 & 1.0 & & & & & & & 0. \\
\hline & & & & 1.082 & & & 0.5 & 0.9 & 1. & 1.258 & \\
\hline & & 0.379 & 1.121 & 1.090 & 0.100 & & 1.000 & & & & \\
\hline \multicolumn{12}{|c|}{ butyl propanoate(1)+nonane(2) } \\
\hline & & 0.0 & - & 1.000 & & & 0.5 & 0. & 1 & & \\
\hline & 0.027 & 0.034 & 1.126 & 1.002 & 0.0 & & 0.600 & & 50 & & 90 \\
\hline & & 0.042 & & 1.002 & & & 0.6 & & 1.045 & & \\
\hline & 0.04 & 0.056 & 1.22 & 1.000 & 0.00 & & 0.628 & 0.629 & 1.044 & 1.1 & \\
\hline & 0.048 & 0.065 & 1.223 & 1.001 & 0.0 & & 0.643 & 0.641 & 1.039 & 1.192 & 0.087 \\
\hline & 0.059 & 0.079 & 1.21 & 1.003 & & & 0.6 & 0.645 & 1.041 & & \\
\hline 22.37 & 0.074 & 0.099 & 1.223 & 1.005 & 0.019 & & 0.665 & 0.661 & 1.036 & 1.199 & 0.084 \\
\hline & 0.084 & 0.112 & 1.223 & 1.005 & & & & & 1.032 & & \\
\hline 421.90 & 0.100 & 0.133 & 1.230 & 1.007 & 0.027 & 417.14 & 0.703 & 0.694 & 1.028 & 1.221 & 0.079 \\
\hline & & & & 1.007 & & & & & 1.025 & & \\
\hline 421.29 & 0.137 & 0.178 & 1.220 & 1.011 & 0.037 & 417.15 & 0.739 & 0.726 & 1.023 & 1.244 & 0.074 \\
\hline & 0.202 & 0.253 & 1.20 & 1.019 & & & 0.757 & 0.742 & 1.020 & & 0.071 \\
\hline & 0.225 & 0.278 & 1.199 & 1.023 & 0.058 & 417.19 & 0.773 & 0.756 & 1.017 & 1.273 & 0.068 \\
\hline & 0.244 & 0.296 & & 1.028 & & & & 0.789 & 1.014 & 1.301 & 0.062 \\
\hline 419.70 & 0.252 & 0.304 & 1.179 & 1.029 & 0.063 & 417.30 & 0.823 & 0.804 & 1.013 & 1.309 & 0.058 \\
\hline
\end{tabular}




\begin{tabular}{|c|c|c|c|c|c|c|c|c|c|c|}
\hline & 0.286 & 0.338 & $1.10 /$ & 1.036 & 0.069 & 417.350 .841 & 0.821 & 1.011 & 1.329 & \\
\hline 419.08 & 0.307 & 0.357 & 1.154 & 1.043 & 0.073 & $\begin{array}{lll}417.47 & 0.858\end{array}$ & 0.837 & 1.007 & 1.352 & 0.049 \\
\hline 418.96 & 0.324 & 0.375 & 1.152 & 1.042 & 0.074 & $417.56 \quad 0.872$ & 0.853 & 1.007 & 1.349 & 0.045 \\
\hline & 0.347 & 0.396 & 1.143 & 1.049 & 0.078 & 417.660 .890 & 0.871 & 1.005 & 1.375 & 0.039 \\
\hline 18.55 & 370 & 0.417 & 1.134 & 1.055 & & 417.790 .906 & 0.889 & 1.004 & 1.380 & 0.034 \\
\hline 418.34 & 0.400 & 0.441 & 1.115 & 1.068 & 0.083 & $\begin{array}{ll}7.87 & 0.920\end{array}$ & 0.902 & 1.001 & 1.429 & 0.030 \\
\hline+17.94 & 0.450 & 0.484 & 1.099 & 1.087 & 0.088 & $\begin{array}{ll}8.01 & 0.937\end{array}$ & 0.922 & 1.001 & 1.439 & 0.024 \\
\hline 417.69 & 0.497 & 0.520 & 1.076 & 1.113 & 0.090 & 8.130 .951 & 0.939 & 1.001 & 1.443 & 0.019 \\
\hline 417.55 & 0.517 & .537 & 1.072 & 1.122 & 0.091 & 8.210 .962 & 0.952 & 1.001 & 1.462 & 0.016 \\
\hline 17.48 & 0.534 & 0.551 & 1.066 & 1.130 & 0.091 & .240 .969 & 0.960 & 1.002 & 1.492 & 0.014 \\
\hline 17.42 & 0.552 & 0.566 & 1.061 & 1.138 & 0.091 & $.40 \quad 0.984$ & 0.979 & 1.002 & 1.512 & 0.008 \\
\hline 17.35 & 0.569 & 0.580 & 1.057 & 1.147 & & $418.70 \quad 1.000$ & 1.000 & 1.000 & - & 0.000 \\
\hline \multicolumn{11}{|c|}{ Run 2} \\
\hline \multicolumn{11}{|c|}{ propyl propanoate(1)+octane(2) (Run 2) } \\
\hline 398.82 & 0.000 & 0.000 & - & 1.000 & 0.000 & 393.280 .367 & 0.428 & 1.237 & 1.048 & 0.108 \\
\hline 398.28 & 0.023 & 0.041 & 1.650 & 0.995 & 0.007 & 3.120 .388 & 0.446 & 1.226 & 1.054 & 0.111 \\
\hline 398.07 & 0.030 & 0.052 & 1.614 & 0.997 & 0.011 & 010.4 & 0.461 & 1.210 & 1.062 & 0.113 \\
\hline 397.8 & 0.039 & 0.066 & 1.583 & 0.997 & 0.015 & 2.960 .416 & 0.468 & 1.203 & 1.066 & 0.114 \\
\hline 397.50 & 0.049 & 0.083 & 1.603 & 0.998 & 0.021 & 2.910 .425 & 0.476 & 1.200 & 1.068 & 0.115 \\
\hline 397. & 0.058 & 0.095 & 1.548 & 0.999 & 0.025 & 790.4 & 0.491 & 1.185 & 1.078 & 118 \\
\hline 397.08 & 0.069 & 0.110 & 1.520 & 1.001 & 0.030 & $392.70 \quad 0.473$ & 0.510 & 1.161 & 1.096 & 0.119 \\
\hline 396.84 & 0.08 & 0.127 & 1.507 & 1.001 & 0.034 & 570.5 & 0.539 & 1.144 & 1.111 & 0.120 \\
\hline 396.59 & 0.094 & 0.146 & 1.502 & 1.000 & 0.038 & 392.490 .549 & 0.569 & 1.124 & 1.132 & 0.120 \\
\hline 396.35 & 0.106 & 0.163 & 1.496 & 0.999 & 0.042 & 410.573 & 0.589 & 1.117 & 1.143 & 0.120 \\
\hline 396.11 & 0.120 & 0.180 & 1.471 & 1.001 & 0.047 & 2.410 .599 & 0.610 & 1.106 & 1.156 & 0.118 \\
\hline 395.85 & 0.137 & 0.202 & 1.457 & 1.000 & 0.052 & .420 .625 & 0.630 & 1.095 & 1.171 & 0.116 \\
\hline 395.55 & 0.15 & 0.228 & 1.466 & 0.996 & 0.056 & 2.490 .699 & 0.689 & 1.068 & 1.226 & 0.107 \\
\hline 395.25 & 0.176 & 0.250 & 1.428 & 1.000 & 0.063 & 392.650 .750 & 0.730 & 1.050 & 1.274 & 0.097 \\
\hline 394.95 & & 0.274 & 1.421 & & 0.068 & 2.860 .797 & 0.770 & 1.036 & 1.330 & 0.086 \\
\hline 394.65 & 0.217 & 0.295 & 1.386 & 1.006 & 0.076 & 3.190 .843 & 0.815 & 1.027 & 1.371 & 0.072 \\
\hline 394.39 & 0.237 & 0.317 & 1.375 & 1.007 & 0.081 & 3.460 .875 & 0.847 & 1.020 & 1.418 & 0.061 \\
\hline 394.15 & 0.259 & 0.339 & 1.356 & 1.010 & 0.086 & 3.950 .921 & 0.897 & 1.013 & 1.487 & 0.043 \\
\hline 393.94 & 0.285 & 0.362 & 1.321 & 1.017 & 0.091 & 4.340 .951 & 0.933 & 1.009 & 1.536 & 0.030 \\
\hline 393.7 & 0.31 & 0.381 & 1.283 & 1.028 & 0.097 & $4.70 \quad 0.973$ & 0.962 & 1.007 & 1.582 & 0.019 \\
\hline 393.57 & 0.330 & 0.398 & 1.267 & 1.034 & 0.101 & 395.150 .991 & 0.987 & 1.002 & 1.596 & 0.006 \\
\hline 393.47 & 0.343 & 0.409 & 1.257 & 1.038 & 0.103 & $418.70 \quad 1.000$ & 1.000 & 1.000 & - & 0.000 \\
\hline \multicolumn{11}{|c|}{ butyl propanoate(1)+octane(2) (Run 2) } \\
\hline 398.82 & 0.000 & 0.000 & - & 1.000 & 0.000 & $407.80 \quad 0.677$ & 0.517 & 1.028 & 1.190 & 0.075 \\
\hline 398.94 & 0.020 & 0.016 & 1.360 & 1.001 & 0.007 & $408.83 \quad 0.724$ & 0.565 & 1.020 & 1.222 & 0.070 \\
\hline 399.20 & 0.042 & 0.033 & 1.348 & 1.000 & 0.012 & 408.420 .702 & 0.544 & 1.024 & 1.200 & 0.071 \\
\hline 399.43 & 0.071 & 0.054 & 1.313 & 1.002 & 0.021 & $409.37 \quad 0.744$ & 0.588 & 1.017 & 1.233 & 0.066 \\
\hline 399.65 & 0.102 & 0.077 & 1.295 & 1.005 & 0.031 & $409.83 \quad 0.762$ & 0.608 & 1.013 & 1.250 & 0.063 \\
\hline 399.99 & 0.143 & 0.107 & 1.268 & 1.010 & 0.042 & $410.57 \quad 0.787$ & 0.641 & 1.013 & 1.255 & 0.059 \\
\hline 400.38 & 0.191 & 0.140 & 1.225 & 1.020 & 0.055 & 411.250 .809 & 0.667 & 1.007 & 1.276 & 0.052 \\
\hline 400.83 & 0.237 & 0.172 & 1.196 & 1.030 & 0.065 & $411.98 \quad 0.832$ & 0.699 & 1.005 & 1.293 & 0.047 \\
\hline 401.26 & 0.279 & 0.200 & 1.167 & 1.041 & 0.072 & $412.80 \quad 0.857$ & 0.733 & 1.001 & 1.317 & 0.040 \\
\hline
\end{tabular}




\begin{tabular}{|llllllllllll|}
\hline 401.73 & 0.318 & 0.228 & 1.151 & 1.050 & 0.078 & 413.49 & 0.874 & 0.763 & 1.002 & 1.307 & 0.036 \\
402.24 & 0.354 & 0.250 & 1.116 & 1.063 & 0.078 & 414.23 & 0.895 & 0.794 & 0.999 & 1.337 & 0.029 \\
402.70 & 0.392 & 0.278 & 1.107 & 1.074 & 0.083 & 414.66 & 0.907 & 0.812 & 0.996 & 1.359 & 0.025 \\
403.19 & 0.426 & 0.303 & 1.095 & 1.084 & 0.085 & 415.15 & 0.919 & 0.836 & 0.999 & 1.351 & 0.023 \\
403.61 & 0.456 & 0.324 & 1.077 & 1.100 & 0.085 & 415.45 & 0.927 & 0.848 & 0.997 & 1.375 & 0.020 \\
404.12 & 0.490 & 0.348 & 1.061 & 1.116 & 0.085 & 416.05 & 0.940 & 0.873 & 0.996 & 1.376 & 0.015 \\
404.50 & 0.516 & 0.366 & 1.049 & 1.132 & 0.085 & 416.55 & 0.950 & 0.894 & 0.995 & 1.366 & 0.011 \\
405.09 & 0.548 & 0.393 & 1.044 & 1.143 & 0.084 & 417.16 & 0.965 & 0.924 & 0.997 & 1.365 & 0.008 \\
405.63 & 0.574 & 0.418 & 1.044 & 1.147 & 0.083 & 417.51 & 0.971 & 0.939 & 0.997 & 1.340 & 0.005 \\
406.16 & 0.600 & 0.443 & 1.041 & 1.155 & 0.082 & 417.87 & 0.981 & 0.957 & 0.996 & 1.419 & 0.003 \\
406.73 & 0.629 & 0.469 & 1.034 & 1.170 & 0.079 & 418.30 & 0.990 & 0.978 & 0.997 & 1.364 & 0.000 \\
407.30 & 0.653 & 0.494 & 1.033 & 1.175 & 0.077 & 418.70 & 1.000 & 1.000 & 1.000 & - & 0.000 \\
\hline
\end{tabular}



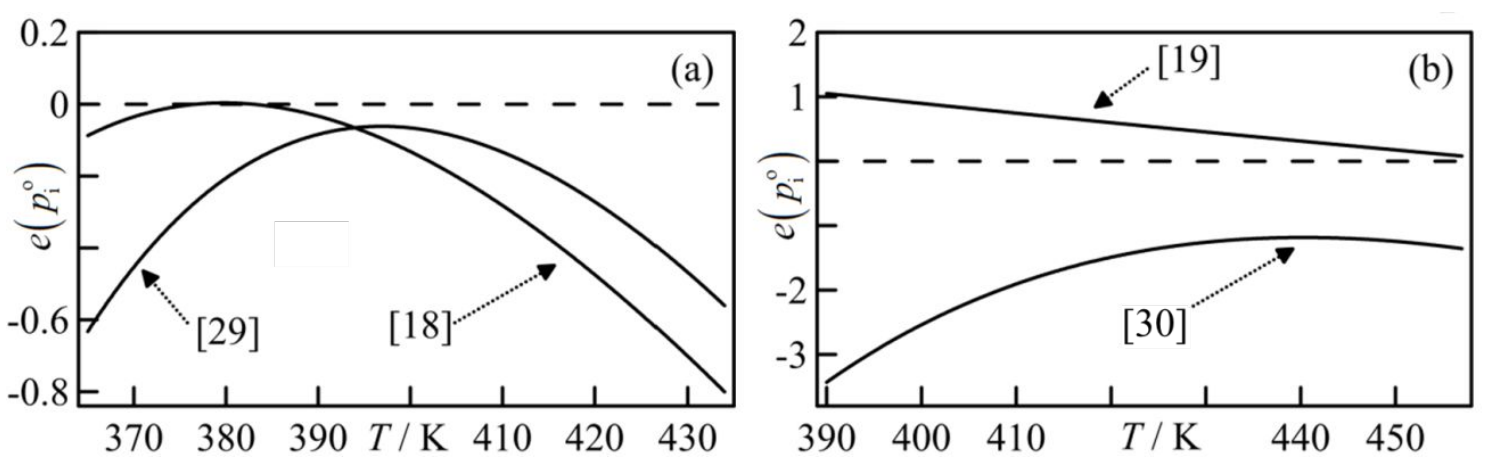

Figure S1. Comparison of errors for vapor pressures: $e\left(p_{\mathrm{i}}^{\mathrm{o}}\right)=100\left(p_{\mathrm{i}, \mathrm{exp}}^{\mathrm{o}}-p_{\mathrm{i}, \mathrm{lit}}^{\mathrm{o}}\right) / p_{\mathrm{i}, \mathrm{exp}}^{\mathrm{o}}$ between the values estimated by Eq 2 (with parameters of Table 1) and those from literature indicated, for: (a) Propyl propanoate, (b) butyl propanoate.. 

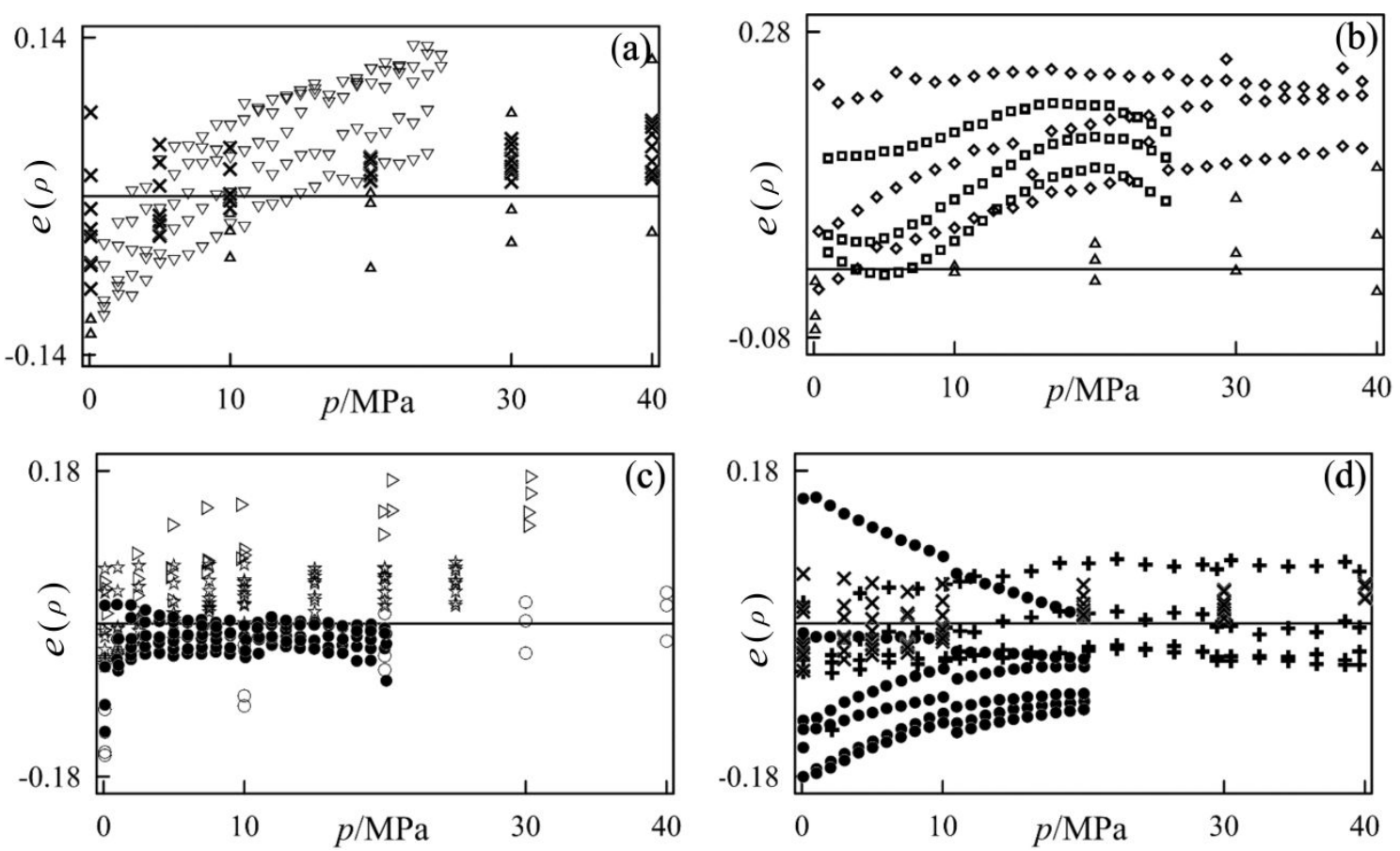

Figure S2. Comparison between experimental $(p, \rho, T)$ values and those from literature for alkanes: (a) hexane, (b) heptane, (c) octane and (d) nonane. $(\triangle) \operatorname{Ref}[34],(\nabla) \operatorname{Ref}$ [35], (x) $\operatorname{Ref}[36],(\diamond) \operatorname{Ref}[37],(\square) \operatorname{Ref}[38],(\triangleright) \operatorname{Ref}[39],(0) \operatorname{Ref}[40],(\diamond) \operatorname{Ref}[41]$, (•) $\operatorname{Ref}[42],(+) \operatorname{Ref}[43]$.

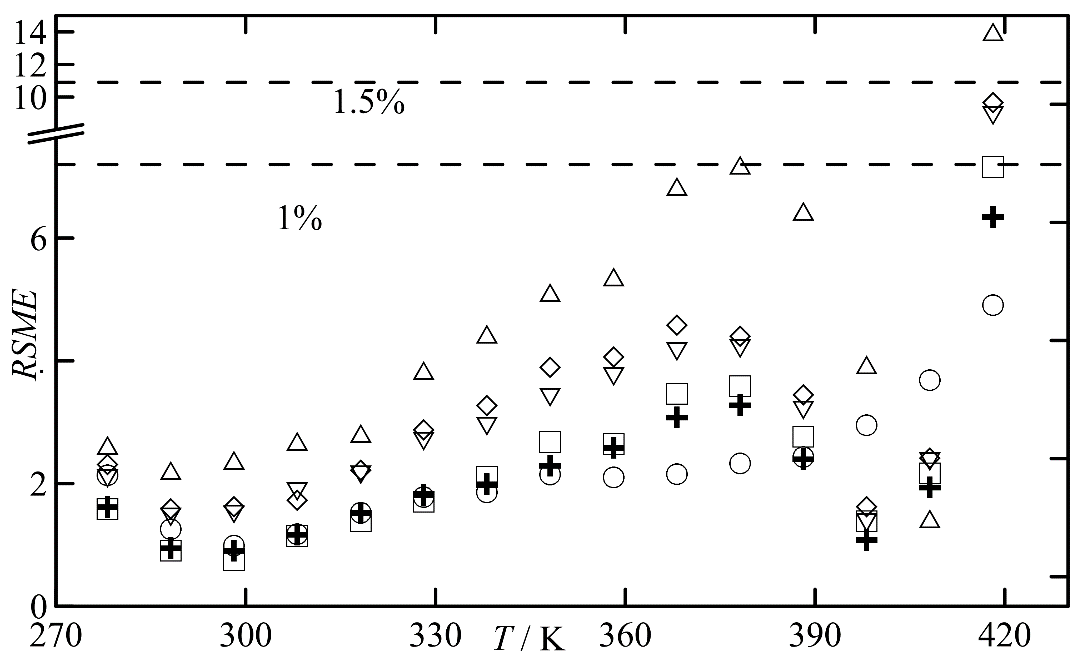

Figure S3. RSME between the densities estimated by Tait equation, eq $\mathrm{A} 1$, and the experimental data of $p-\rho-T$. Symbols indicate the compound: $(O)$ hexane, $(\Delta)$ heptane, $(\nabla)$ octane, $(\mathbf{+})$ nonane, $(\diamond)$ propyl propanoate, $(\square)$ butyl propanoate. 

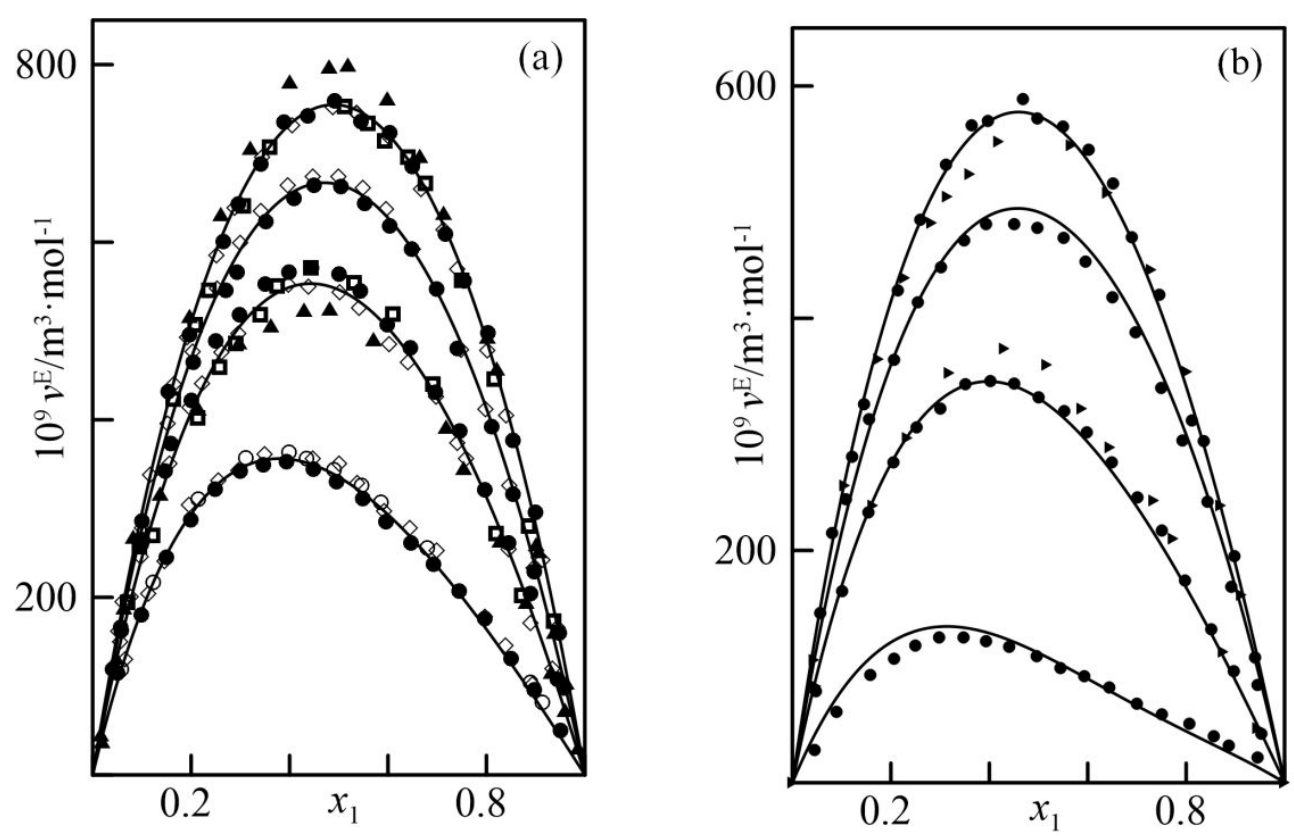

Figure S4. Comparison between experimental excess molar volumes $\left(x_{1}, v^{\mathrm{E}}\right)$ measured at $298.15 \mathrm{~K}$ and values from literature for: (a) propyl propanoate(1)+alkane(2), (b) butyl propanoate(1)+alkane(2). Labels indicate the number of carbon atoms of hydrocarbon. (๑) Experimental values; solid-line: modeling results. (O) Ref [1], ( $\diamond) \operatorname{Ref}[2],(\mathbf{\Delta}) \operatorname{Ref}$

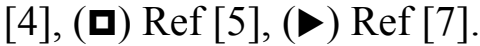
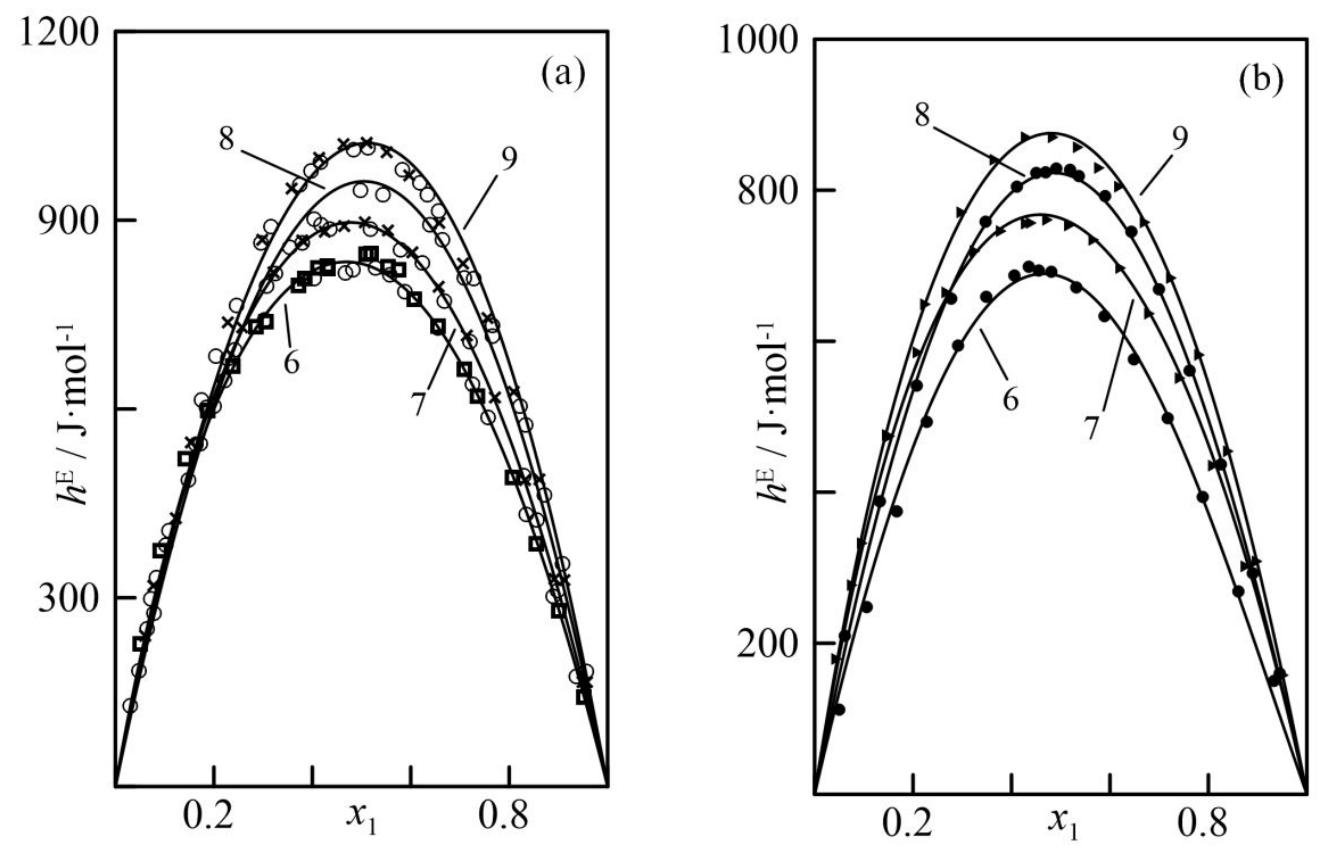

Figure S5. Experimental excess molar enthalpies $\left(x_{1}, h^{\mathrm{E}}\right)$ obtained at $298.15 \mathrm{~K}$ and comparison with literature values used in modelling for: (a) propyl propanoate(1)+ alkane(2), (b) butyl propanoate(1)+alkane(2). Labels indicate the number of carbon atoms of hydrocarbon. (๑) Experimental values, solid-line: modeling results, (O) Ref [2], (ㅁ) Ref [3], (x) Ref [5], ( $)$ Ref. [7]. 

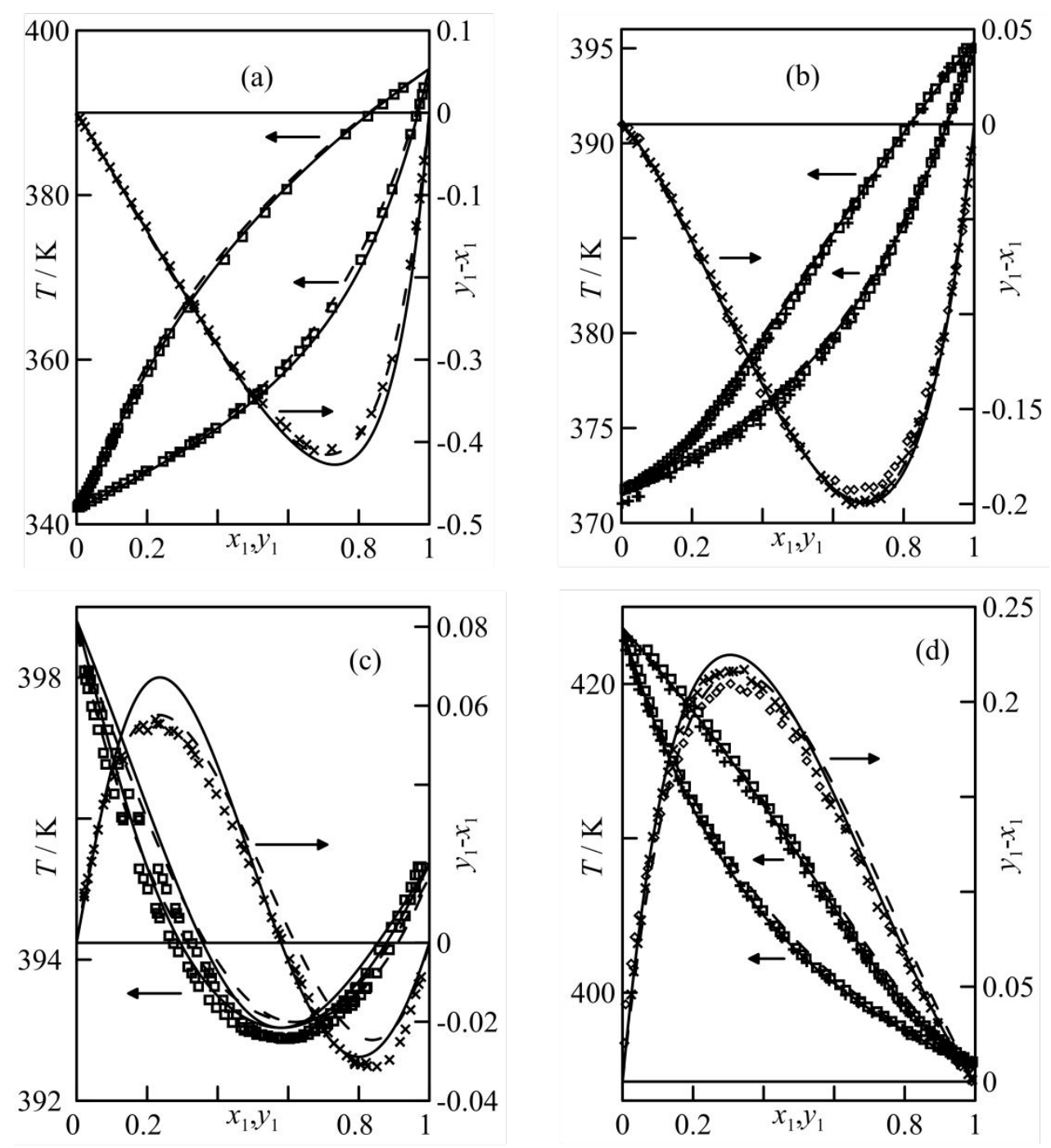

Figure S6. Representation of iso- $p$ VLE experimental data of $T$ vs $x_{1}, y_{1}$ (Run 1: $\square$ ) and $y_{1}-x_{1}$ vs $x_{1}$ (Run 1: $\mathbf{X}$ ), for the binaries formed by propyl propanoate(1): (a) thexane, (b) theptane, (c) +octane, (d) +nonane. Solid-lines (_- correspond to modeling, dashedlines (-- ) UNIFAC [52]. (+) and $(\diamond)$ are, respectively, values of $T$ vs $x_{1}, y_{1}$ and $y_{1}-x_{1}$ vs $x_{1}$ from ref. [4]. 

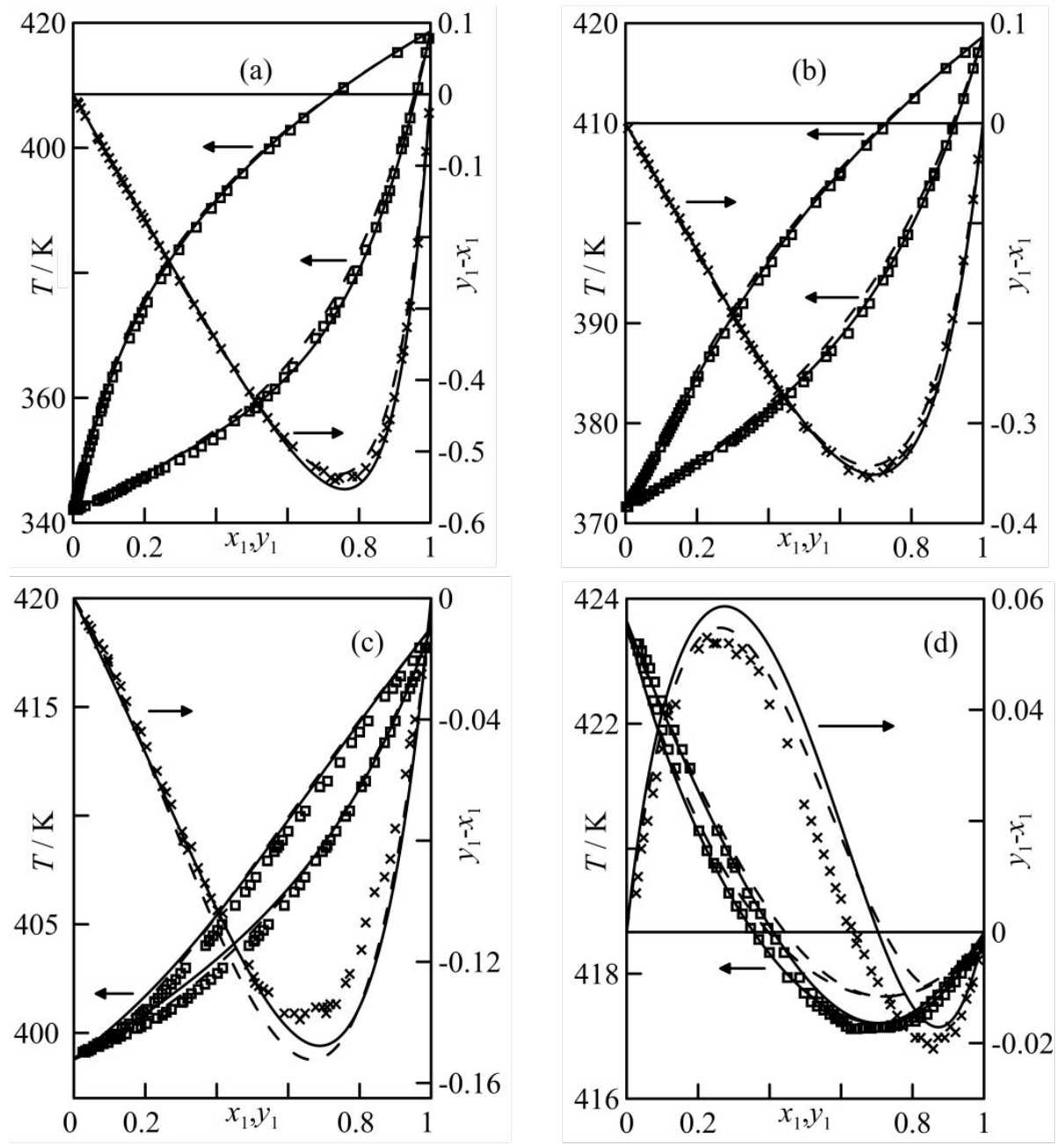

Figure S7. Representation of iso- $p$ VLE experimental data of $T$ vs $x_{1}, y_{1}$ (Run 1: $\mathbf{\square}$ ) and $y_{1}-x_{1}$ vs $x_{1}$ (Run 1: $\mathbf{x}$ ), for the binaries formed by butyl propanoate(1): (a) thexane, (b) +heptane, (c) +octane, (d) + nonane. Solid-lines (- correspond to modeling, dashedlines (- - ) UNIFAC [52]. 